\title{
Impact of acquisition and analysis strategies on cortical depth-dependent fMRI
}

Citation for published version (APA):

Kashyap, S., Ivanov, D., Havlicek, M., Poser, B. A., \& Uludağ, K. (2018). Impact of acquisition and analysis strategies on cortical depth-dependent fMRI. Neuroimage, 168, 332-344.

https://doi.org/10.1016/j.neuroimage.2017.05.022

Document status and date:

Published: 01/03/2018

DOI:

10.1016/j.neuroimage.2017.05.022

Document Version:

Publisher's PDF, also known as Version of record

Document license:

Taverne

Please check the document version of this publication:

- A submitted manuscript is the version of the article upon submission and before peer-review. There can be important differences between the submitted version and the official published version of record.

People interested in the research are advised to contact the author for the final version of the publication, or visit the DOI to the publisher's website.

- The final author version and the galley proof are versions of the publication after peer review.

- The final published version features the final layout of the paper including the volume, issue and page numbers.

Link to publication

\footnotetext{
General rights rights.

- You may freely distribute the URL identifying the publication in the public portal. please follow below link for the End User Agreement:

www.umlib.nl/taverne-license

Take down policy

If you believe that this document breaches copyright please contact us at:

repository@maastrichtuniversity.nl

providing details and we will investigate your claim.
}

Copyright and moral rights for the publications made accessible in the public portal are retained by the authors and/or other copyright owners and it is a condition of accessing publications that users recognise and abide by the legal requirements associated with these

- Users may download and print one copy of any publication from the public portal for the purpose of private study or research.

- You may not further distribute the material or use it for any profit-making activity or commercial gain

If the publication is distributed under the terms of Article $25 \mathrm{fa}$ of the Dutch Copyright Act, indicated by the "Taverne" license above, 


\title{
Impact of acquisition and analysis strategies on cortical depth-dependent fMRI
}

\author{
Sriranga Kashyap*, Dimo Ivanov, Martin Havlicek, Benedikt A. Poser, Kâmil Uludağ \\ Department of Cognitive Neuroscience, Faculty of Psychology and Neuroscience, Maastricht University, Maastricht, The Netherlands
}

\section{A R T I C L E I N F O}

\section{Keywords:}

Ultra-high field

High resolution

Distortion-matched $\mathrm{T}_{1}$ anatomy

Cortical depth-dependent fMRI

\begin{abstract}
A B S T R A C T
Functional MRI at ultra-high magnetic fields $(\geq 7 \mathrm{~T}$ ) provides the opportunity to probe columnar and laminar processing in the human brain in vivo at sub-millimeter spatial scales. However, fMRI data only indirectly reflects the neuronal laminar profile due to a bias to ascending and pial veins inherent in gradient- and spinecho BOLD fMRI. In addition, accurate delineation of the cortical depths is difficult, due to the relatively large voxel sizes and lack of sufficient tissue contrast in the functional images. In conventional depth-dependent fMRI studies, anatomical and functional data are acquired with different image read-out modules, the fMRI data are distortion-corrected and vascular biases are accounted for by subtracting the depth-dependent activation profiles of different stimulus conditions. In this study, using high-resolution gradient-echo fMRI data $(0.7 \mathrm{~mm}$ isotropic) of the human visual cortex, we propose instead, that depth-dependent functional information is best preserved if data analysis is performed in the original functional data space. To achieve this, we acquired anatomical images with high tissue contrast and similar distortion to the functional images using multiple inversion-recovery time EPI, thereby eliminating the need to un-distort the fMRI data. We demonstrate higher spatial accuracy for the cortical layer definitions of this approach as compared to the more conventional approach using MP2RAGE anatomy. In addition, we provide theoretical arguments and empirical evidence that vascular biases can be better accounted for using division instead of subtraction of the depth-dependent profiles. Finally, we show that the hemodynamic response of grey matter has relatively stronger post-stimulus undershoot than the pial vein voxels. In summary, we show that the choice of fMRI data acquisition and processing can impact observable differences in the cortical depth profiles and present evidence that cortical depth-dependent modulation of the BOLD signal can be resolved using gradient-echo imaging.
\end{abstract}

\section{Introduction}

Functional magnetic resonance imaging (fMRI) has typically been utilized for localizing brain areas involved in processing cognitive and/ or sensory tasks or in resting-state activity (recent overviews, see Uludağ et al. (2015)). The spatial resolutions of these mapping studies are usually between $8 \mathrm{~mm}^{3}$ and $64 \mathrm{~mm}^{3}$ (i.e. $2-4 \mathrm{~mm}$ along each voxel dimension). Given that the human cortex is about $3 \mathrm{~mm}$ thick (Brodmann, 1909; von Economo, 1929), studying neuronal processing within the six cellular layers of the cortex is usually not feasible at field strengths of $3 \mathrm{~T}$ and below (but see Koopmans et al. (2010); Ress et al. (2007)). With the advent of ultra-high magnetic field (UHF) human MRI scanners, i.e., $7 \mathrm{~T}$ and above, early studies have achieved spatial resolutions below $1 \mathrm{~mm}$ in-plane and above $1 \mathrm{~mm}$ slice thickness (Pfeuffer et al., 2002; Yacoub et al., 2008) and, recently, several studies have been published utilizing sub-millimeter isotropic voxels (De Martino et al., 2015; De Martino et al., 2013; Fracasso et al., 2017;
Huber et al., 2016a; Kemper et al., 2015; Kok et al., 2016; Muckli et al., 2015; Olman et al., 2012; Zimmermann et al., 2011). In the context of cortical depth-dependent fMRI, high-resolution imaging at UHF is crucial to reduce partial voluming of grey matter (GM) voxels with white matter (WM) and cerebrospinal fluid (CSF) (Hoogenraad et al., 1999; Koopmans et al., 2011; Logothetis et al., 2002; Polimeni et al., 2010a; Ress et al., 2007; Yacoub et al., 2003), leading to a paradigm shift in the type of neuroscientific questions that can be investigated in vivo in humans. Recent studies have probed the columnar and laminar organization of the cortex (Douglas and Martin, 2004), previously only possible in animal models, by acquiring not only the anatomical but also the functional MRI in humans at a sub-millimeter scale (e.g. De Martino et al. (2015); De Martino et al. (2013); Fracasso et al. (2017); Kemper et al. (2015); Kok et al. (2016); Muckli et al. (2015); Olman et al. (2012); Zimmermann et al. (2011)). Please note, the term fMRI will refer to blood oxygenation level-dependent (BOLD) (Ogawa et al., 1990a; Ogawa et al., 1990b) fMRI using gradient echo, unless other

\footnotetext{
* Corresponding authors.

E-mail addresses: sriranga.kashyap@maastrichtuniversity.nl (S. Kashyap), kamil.uludag@maastrichtuniversity.nl (K. Uludağ).
} 
functional contrasts, such as cerebral blood volume (CBV) (Mandeville et al., 1998) or cerebral blood flow (CBF) (Kwong et al., 1992), are explicitly mentioned.

In addition to the increased accessibility to UHF scanners, advances in MRI technology such as RF-coil technology (recent overviews, see Vaughan and Griffiths (2012) and references therein), parallel imaging techniques (Griswold et al. (2002); Pruessmann et al. (1999) and recent overview, Poser and Setsompop, 2017) and optimizations of multi-modal MRI sequences (Huber et al., 2016b; Ivanov et al., 2016) have enabled researchers to push the spatial and temporal boundaries of fMRI. fMRI at UHF takes advantage of the substantial gain in image and temporal signal-to-noise (SNR) (Vaughan et al., 2001), increased microvascular BOLD signal for both gradient-echo (GE) and spin-echo (SE) contrasts (Uludag et al., 2009) and sub-millimeter spatial resolution to better localize the hemodynamic signal in the cortex (Ugurbil et al., 2003a). Nevertheless, both the acquisition and analysis of such high-resolution data are still being developed and optimized to unravel depth-dependent fMRI signals.

fMRI acquisitions probe neuronal activity indirectly via induced vascular changes and it has been experimentally and numerically shown that both GE and SE have contributions from surface pial and intra-cortical ascending veins (see Uludag et al. (2009) and references therein). A dynamic biophysical model is needed to remove the vascular bias present in GE and SE data and successfully disentangle the neural from the vascular contributions. Modeling efforts are underway to address this issue in the context of depth-dependent fMRI (Heinzle et al., 2016; Markuerkiaga et al., 2016). In the absence of a comprehensive model-driven approach, differences in the fMRI signal between stimulus conditions (i.e. subtraction) have been considered to faithfully represent the underlying electrophysiological profiles (Harel et al., 2006; Kim et al., 2000; Logothetis, 2008; Mathiesen et al., 1998; Olman et al., 2012; Ugurbil et al., 2003b).

In depth-dependent GE-fMRI, there are two main vascular biases, a) draining of deoxygenated hemoglobin $(\mathrm{dHb})$ from lower to upper layers of the cortex via ascending veins, and b) baseline venous CBV $\left(\mathrm{V}_{0}\right)$ and relaxation parameters, such as $\mathrm{T}_{2}{ }^{*}$, which can be different at the different depths. The first bias (draining $\mathrm{dHb}$ ) is approximately an additive component to the local microvasculature signal (see Uludag et al. (2009)). That is, the total BOLD signal of a given layer consists of the local microvasculature- and the ascending veins-signals. The second bias (combination of $\mathrm{V}_{0}$ and other layer-specific baseline parameters) is an indicator for the layer's sensitivity for activation, i.e. the higher the baseline $\mathrm{CBV}$ value, the higher is the amount of $\mathrm{dHb}$ in that layer and the higher is the BOLD signal for the same neuronal activity changes. Here, we propose to reduce the latter bias by dividing depth-dependent signals between conditions and/or time points (Uludag and Blinder, 2017). Below, we provide a detailed theoretical justification of the "division" approach and provide evidence that division, rather than subtraction, better accounts for the vascular biases in the GE fMRI signal.

fMRI data, usually acquired with an echo-planar imaging (EPI) read-out, is strongly affected by local variations in magnetic susceptibility (e.g. at tissue-air interface) resulting in geometric distortions (Jezzard and Clare, 1999), which are most pronounced in the phaseencoding direction. In contrast, the anatomical data, typically acquired with a $\mathrm{T}_{1}$-weighting using a Magnetization Prepared RApid GradientEcho (MPRAGE) (Mugler and Brookeman, 1990), or with additional quantitative $\mathrm{T}_{1}$ using a Magnetization Prepared 2 Rapid Acquisition Gradient Echoes (MP2RAGE) (Marques et al., 2010), have comparatively lesser distortions. The main reason to utilize a $\mathrm{T}_{1}$ anatomy for cortical layer definitions is because of the higher tissue contrast between GM, WM and CSF compared to EPI-fMRI data. Therefore, most depth-dependent BOLD fMRI studies so far have relied on the conventional acquisition approach using a differently-distorted structural and functional datasets, such as: MEMPRAGE \& EPI (Polimeni et al., 2010a), MPRAGE \& 3D-GRASE (De Martino et al., 2015;
Muckli et al., 2015; Olman et al., 2012; Zimmermann et al., 2011), MPRAGE \& EPI (Fracasso et al., 2017; Muckli et al., 2015; Nasr et al., 2016), or MP2RAGE \& EPI (Kok et al., 2016). Alternatively, depthdependent functional data has been acquired distortion-matched to the MPRAGE (Koopmans et al., 2011) using a GRE 3D-FLASH readout but at the cost of much lower fMRI temporal resolution compared to an EPI acquisition. Therefore, similar to low spatial resolution studies, the standard high-resolution neuroimaging dataset for depth-dependent fMRI also consists of functional and anatomical data acquired with different pulse sequences, encoding schemes and readouts. Consequently, the different geometric distortions pose limitations on achieving accurate co-registration which is critical in studies probing the cortical micro-circuitry using high-resolution depth-dependent fMRI. Several techniques have been developed to "distortion-correct" the functional data, thereby, making it geometrically similar to the anatomical reference (Jezzard (2012), and references therein). Commonly used methods for distortion-correction (apart from nonlinear image registration) use a $\mathrm{B}_{0}$ field map-based (Jezzard and Balaban, 1995) or opposite phase-encoded EPI image-based (Andersson et al., 2003) unwarping of the functional data (Fritz et al., 2014). Limitations of distortion-correction methods, such as blurring of the signal due to smoothing, loss in effective spatial resolution due to sub-voxel shifts (Hutton et al., 2002), reduced fidelity of the fMRI due to incomplete recovery of spatial information in distorted regions (Munger et al., 2000) and imperfect distortioncorrection can additionally result in registration errors with the anatomical reference.

One of the outstanding problems for depth-dependent fMRI is the spatially accurate anatomical definition of the cortical depths (or laminae). For the purposes of accurate anatomical definition of laminae, the anatomical image can be distorted either through postprocessing or by modifying the acquisition. For example, EPI, with an identical readout as the functional data, has been described to be a suitable method for fast $\mathrm{T}_{1}$ mapping using an inversion recovery preparation (Clare and Jezzard, 2001; Gowland and Mansfield, 1993; Mansfield et al., 1986; Ordidge et al., 1990; Stehling et al., 1990; Stehling et al., 1991) for anatomical imaging at field strengths $\leq 3 \mathrm{~T}$ and recently, with multiple inversion-recovery time EPI (MI-EPI) at $7 \mathrm{~T}$ (Renvall et al., 2016). Contrary to the conventional data acquisition approaches, using MI-EPI can yield high tissue contrast similar to MP2RAGE but with the advantage of being distortion-matched to the fMRI data. Since high accuracy registration and minimal post-processing of the functional data is of paramount importance for depthdependent fMRI studies, a clear advantage of the MI-EPI approach is the opportunity to forego the need to distortion-correct the fMRI data and potentially achieve higher registration accuracy with the distortion-matched anatomical reference. In addition, this approach limits the potential blurring associated with distortion-correction and hence, the reduction of cortical depth-specific information. Although the MIEPI approach offers a very promising alternative, its performance with respect to depth-dependent fMRI analysis has not yet been quantitatively compared to other acquisition approaches.

The present study compares two workflows for analyzing depthdependent fMRI data, namely, the MI-EPI workflow (analysis in native EPI space i.e. with GE-EPI fMRI and MI-EPI $\mathrm{T}_{1}$ data) to the Conventional workflow (analysis in anatomical space i.e. with distortion-corrected GE-EPI fMRI and MP2RAGE data) for sub-millimeter depth-dependent fMRI at $7 \mathrm{~T}$. We present a processing workflow for depth-dependent analysis of GE-EPI functional data using distortionmatched anatomy acquired with MI-EPI and demonstrate that the MIEPI $\mathrm{T}_{1}$ can be used to accurately define cortical depths in the native EPI space.

In this study, we also propose that the depth-dependent specificity of the GE signal can be improved by normalizing the spatial profile of contrasting stimulus conditions with each other or considering the transients of the hemodynamic response, such as the ratio of the post- 
stimulus undershoot to the positive BOLD signal (Zhao et al., 2007). Although the GE-fMRI signal is limited in its spatial specificity (De Martino et al., 2013; Goense and Logothetis, 2006; Norris, 2012; Uludag et al., 2009; Zhao et al., 2006) due to an inherent bias to draining veins both intra-cortically and on the pial surface, it remains a popular approach to acquire functional data in depth-dependent studies (Fracasso et al., 2017; Kok et al., 2016; Muckli et al., 2015; Nasr et al., 2016; Polimeni et al., 2010a). Since the GE signal change is expected to be the largest along the pial surface and around large veins, we also investigated the dynamics of the event-related average timecourse from CSF and venous voxels. Lastly, given the mesoscopic scale of the cortical circuitry being probed using depth-dependent fMRI, we investigated how the size of the region-of-interest (ROI) affects the sampled depth-dependent GE-EPI signal. In short, this study presents the current state-of-the-art in acquisition and practical considerations for the analysis of high-resolution depth-dependent BOLD fMRI data at ultra-high magnetic fields.

\section{Methods}

Five healthy subjects (two females, median age 28 years) with normal or corrected-to-normal vision participated in this study. Written informed consent was obtained and subjects were duly compensated for their participation. The procedures were conducted with prior approval from the Ethical Committee of the Faculty of Psychology and Neuroscience at Maastricht University.

\section{Experimental paradigm}

Each run consisted of either static or flickering checkerboards as stimuli, interspersed with isoluminant grey screen as resting condition. The experimental runs alternated between static and flickering stimuli. The static (Michelson contrast 1) and flickering $(\sim 8 \mathrm{~Hz}$, Michelson contrast $1 / 3$ ) checkerboard hemi-annuli (see Supplementary Material Fig. 1) were designed based on an earlier study (Sadaghiani et al., 2009) to induce the same positive BOLD (PB) signal amplitude but eliciting a larger post-stimulus undershoot (PSU) for the flickering compared to the static stimulus (Mullinger et al., 2013). The stimuli were created and presented to the left visual hemi-field using PsychoPy (Peirce, 2007) in a block design for $20 \mathrm{~s}$ followed by $40 \mathrm{~s}$ of rest, with each of the six runs consisting of ten blocks of stimulus and rest. The initial baseline resting duration was $40 \mathrm{~s}$, resulting in a total duration of $640 \mathrm{~s}$ for each run. During the scans, subjects were asked to remain motionless and fixate on a central white fixation cross.

\section{Data acquisition}

All data were acquired on a whole-body Magnetom $7 \mathrm{~T}$ research scanner (Siemens Medical Solutions, Erlangen, Germany) using a 32channel phased-array head coil (Nova Medical, Wilmington, MA, USA). Sequence parameters, described below, were optimized in pilot experiments.

\section{Anatomical data}

MP2RAGE: Whole-brain quantitative $\mathrm{T}_{1}$ images were obtained at 0.7 $\mathrm{mm}$ isotropic resolution using a 3D-MP2RAGE sequence (Marques et al., 2010) (240 sagittal slices; GRAPPA=3; partial Fourier=6/8; Ref. $\mathrm{PE}$ lines $=24 ; \mathrm{TR}=5000 \mathrm{~ms} ; \mathrm{TI}_{1} / \mathrm{TI}_{2}=900 / 2750 \mathrm{~ms} ; \alpha_{1} / \alpha_{2}=5^{\circ} / 3^{\circ}$; phase encoding=anterior-posterior) and was followed by a whole-brain $\mathrm{B}_{1}{ }^{+}$map using an Sa2RAGE sequence (Eggenschwiler et al., 2012; Marques and Gruetter, 2013) (TR=2400 ms; $\mathrm{TI}_{1} / \mathrm{TI}_{2}=58 / 1800 \mathrm{~ms} ; \alpha_{1} /$ $\alpha_{2}=4^{\circ} / 10^{\circ}$ ).
MI-EPI: To obtain a $\mathrm{T}_{1}$-anatomy identically distorted as the functional images (see below), an MI-EPI dataset was acquired at 0.7 $\mathrm{mm}$ isotropic (60 oblique coronal slices; GRAPPA=3; partial Fourier=6/8; matrix=214 $\times 214$; fat saturation; $\mathrm{BW}=1016 \mathrm{~Hz} / \mathrm{px}$; Ref. lines $\mathrm{PE}=63$; phase encoding=right-left). 30 inversion times (TI) were acquired with EPI readouts $\left(\mathrm{TI}_{1}=175 \mathrm{~ms}\right.$; slice scan time $=81.75 \mathrm{~ms}$; Excitation flip angle $=73^{\circ}$; echo spacing $=1.09 \mathrm{~ms}$; EPI factor $=214$; $\mathrm{TE}=29 \mathrm{~ms} ; \mathrm{TR}=5107.2 \mathrm{~ms}$; series order=ascending) following a 15 $\mathrm{ms}$ tr-FOCI inversion pulse optimized for an inversion thickness of 200 $\mathrm{mm}$ and the pulse-specific parameters were 3.320 .300 .640 .270 .59 0.001 .007 .713 .900 .250 .40 (see Table 1, Pulse 4, Hurley et al. (2010)). The slice ordering was changed every TR (Clare and Jezzard, 2001) by permuting a slab of slices (in our case, slab-size of 2) so that the slices have different inversion times than in the previous TR (Renvall et al., 2016). Therefore, each run lasted $154 \mathrm{~s}$ consisting of thirty volumes (one for each inversion time, see Supplementary Material Fig. 2) and ten repetitions were acquired. An optimized $\{3 * 2\}$ GRAPPA kernel (Ivanov et al., 2016) was used to reconstruct the images at all TIs. The reference lines were acquired without inversion preparation using FLEET (Polimeni et al., 2016).

\section{Functional data}

BOLD fMRI datasets were acquired for each subject at $0.7 \mathrm{~mm}$ isotropic resolution (52 oblique coronal slices; GRAPPA=3; Flip angle $=16^{\circ}$; partial Fourier 6/8; TR=2940 ms; TE=26 ms; phase encoding=right-left) using 3D GE-EPI (Poser et al., 2010). Ten opposite phase-encoded volumes, with otherwise identical imaging parameters, were acquired after each run to allow for distortioncorrection.

\section{Data processing and analysis}

The processing of the anatomical and functional data was performed using two distinct pipelines, namely, the Conventional and MIEPI workflows, as illustrated in Fig. 1.

\section{Anatomical data}

Conventional workflow: $\mathrm{T}_{1}$-values and GM-WM contrast derived using the MP2RAGE sequence can be affected by $\mathrm{B}_{1}{ }^{+}$transmit inhomogeneities (Marques and Gruetter, 2013). Therefore, the

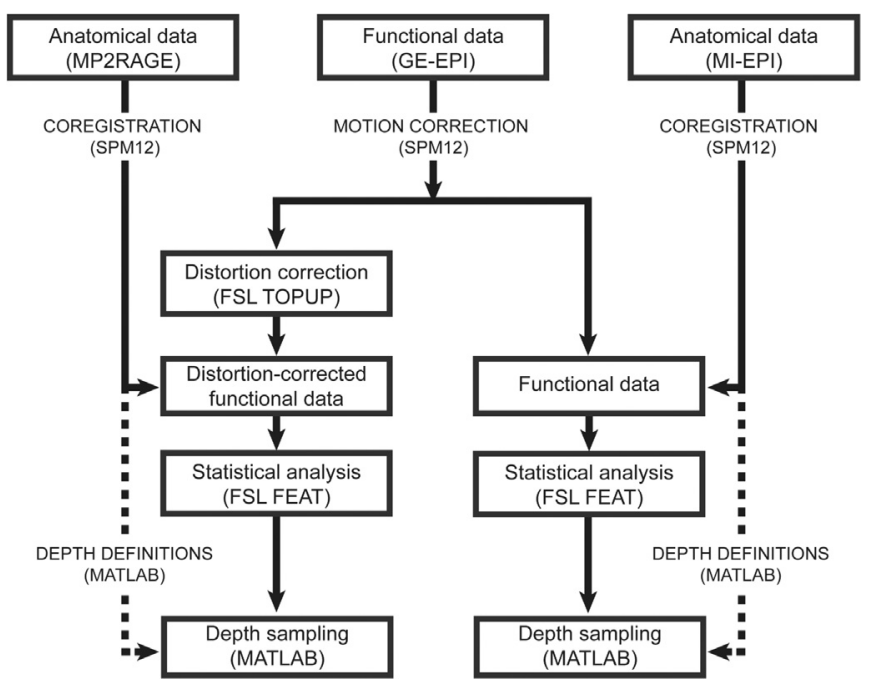

Fig. 1. Data processing and analysis steps in the Conventional workflow (left) and MIEPI workflow (right). Details, please see text. 

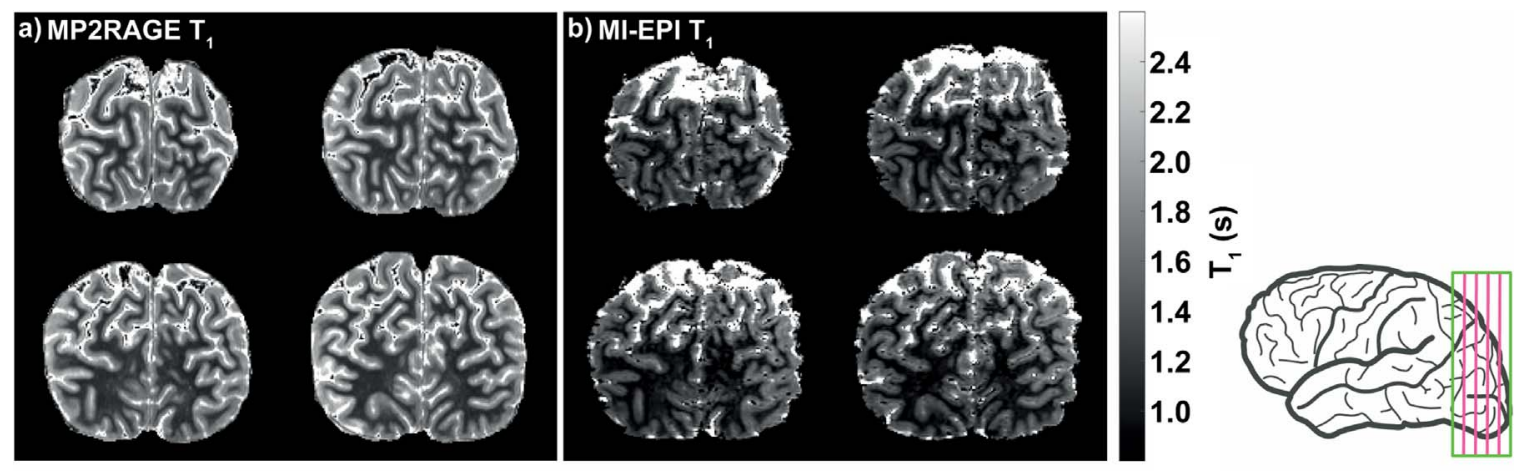

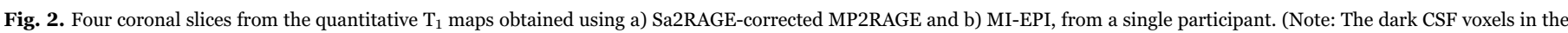
MP2RAGE are an artefact of the $\mathrm{B}_{1}{ }^{+}$-correction.).
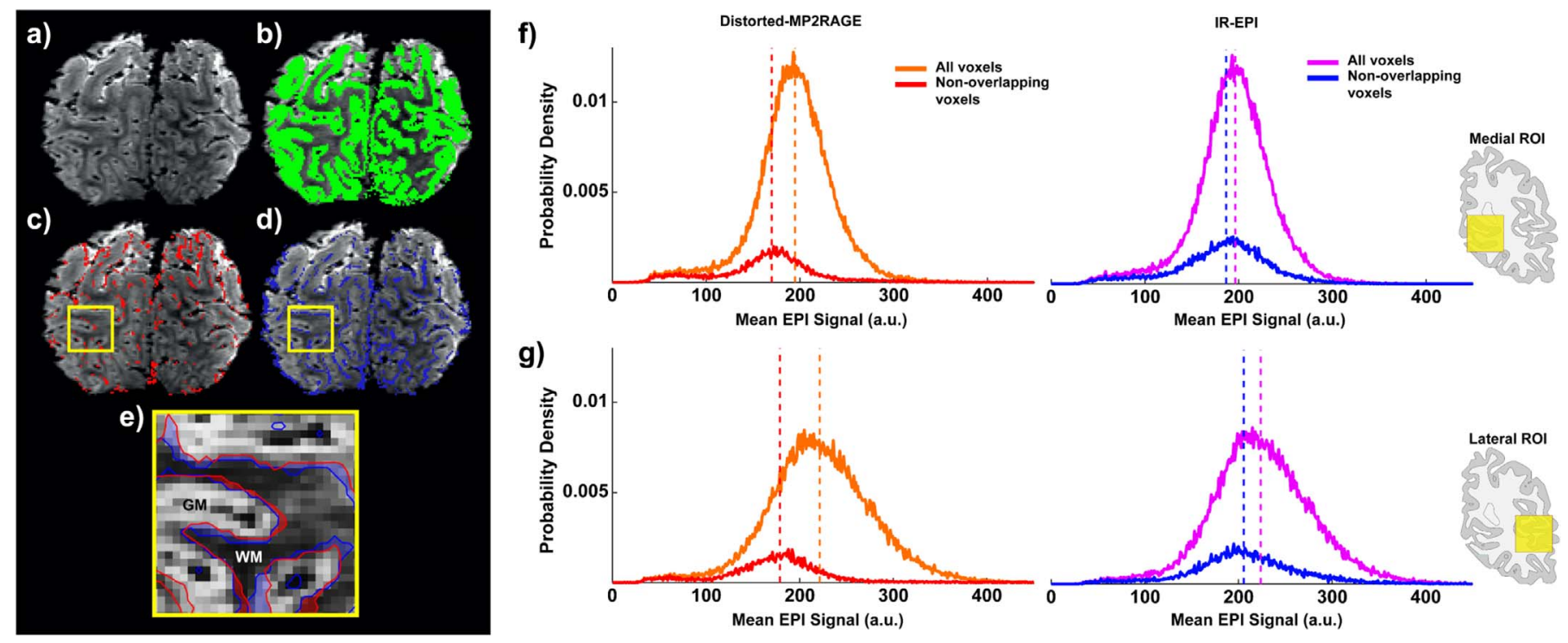

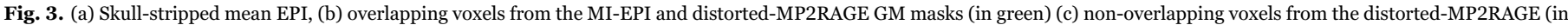

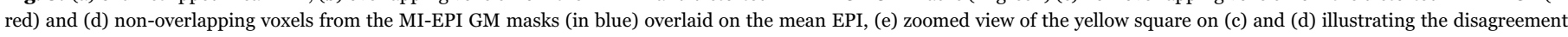

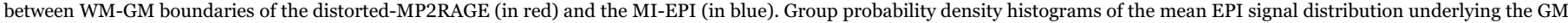

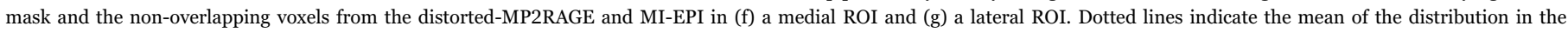
respective color code.

quantitative $\mathrm{T}_{1}$ image of the MP2RAGE was corrected using the Sa2RAGE data (Fig. 2a) and the corrected $\mathrm{T}_{1}$ map was computed in MATLAB (Haast et al., 2016). The whole-brain MP2RAGE was cropped to a field-of-view (FOV) comparable to that of the functional data using the FSL (Smith et al., 2004) function fslroi and reoriented (from sagittal) to correspond with the functional data (coronal) using fslswapdim. The cropping is important as only the anatomical slices relevant for the functional data analysis are considered in the costfunction and the prior reorientation of the MP2RAGE to reduce large rotations during co-registration.

MI-EPI workflow: Ten repetitions of each inversion time of the MIEPI were averaged and a quantitative $\mathrm{T}_{1}$ map (Fig. 2b) was computed offline in MATLAB (MathWorks, USA). The non-linear least squares fitting algorithm based on an adapted version of the three-parameter inversion-recovery model (see Eq. 4, Stikov et al. (2015)), which did not require the exact knowledge of the voxel-specific flip angles, was used to compute the quantitative $\mathrm{T}_{1}$ values.

Segmentation: Initial segmentation was done on the co-registered $\mathrm{T}_{1}$ maps, independently for both the MI-EPI and MP2RAGE data using
SPM12 (Ashburner, 2007; Ashburner and Friston, 2005). The tissue probability maps for GM, WM and CSF were then thresholded ( $>0.5$ ) and binarized into tissue-specific masks. The accuracy of the segmented GM ribbon across all slices in the ROI is very critical for the depth-dependent analysis. Therefore, the tissue-masks were manually corrected using ITK-SNAP v3.4.0 (Yushkevich et al., 2006), in regions where the automatic segmentation was not accurately following the cortical ribbon (e.g., a missing voxel in an otherwise contiguous patch of GM), to obtain an optimum delineation of the GM.

Co-registration analysis: To evaluate the co-registration discrepancy between the Conventional and MI-EPI workflows, we investigated the case where the MP2RAGE is geometrically distorted to closely match the functional data. If distortion-correction were to be optimal in undistorting the functional data into anatomical (MP2RAGE) space, the inverse transformed MP2RAGE using the same voxel displacement estimates should also accurately conform to the native (distorted) EPI space. By sampling from the native EPI data, any discrepancies observed from this approach will most likely reflect errors in registration. The TOPUP voxel shift estimated from the functional data was applied in the opposite direction using FSL's applytopup to the MP2RAGE $\mathrm{T}_{1}$ and its GM mask obtained from its automatic segmentation, thereby distorting them similarly to the mean EPI. 
From the MI-EPI, distorted-MP2RAGE images and their segmentation masks, voxels were classified as either overlapping or non-overlapping voxels. An intersection operation of the MI-EPI and distortedMP2RAGE segmentation masks was first carried out to identify the overlapping GM voxels (Fig. 3b). An exclusive disjunction operation between the overlapping GM mask and the distorted-MP2RAGE GM segmentation was calculated to identify the non-overlapping distortedMP2RAGE GM voxels (Fig. 3c), i.e., voxels that are exclusively GM in the distorted-MP2RAGE mask and not in the MI-EPI mask. The same approach was used to identify the non-overlapping MI-EPI GM voxels (Fig. 3d). Probability density histograms of the mean 3D-EPI signal across the non-overlapping voxels underneath the GM masks were computed for the MI-EPI and the distorted-MP2RAGE in a medial occipital ROI (Fig. 3f, approximately V1) and in a lateral occipital ROI (Fig. 3g, approximately V2/V3) which exhibited different extent of distortions along the phase-encoding direction. It is important to note that this step was solely performed to quantify the quality of the coregistration and was not further used to analyze the functional data in either of the two workflows.

\section{Functional data}

The functional runs were motion-corrected using SPM12 (http:// www.fil.ion.ucl.ac.uk/spm/) and no spatial smoothing was applied. Following this, the functional datasets were analyzed in two independent workflows based on whether distortion-correction was applied (Conventional workflow) or not (MI-EPI workflow). All functional datasets were high-pass filtered in FSL (https://fsl.fmrib. ox.ac.uk/fsl/) using a Gaussian-weighted least-squares fitting ( $\sigma=$ $60 \mathrm{~s}$ ). Statistical analysis of the time-series was carried out in FSL using FEAT v6.0 (FMRI Expert Analysis Tool) using FILM with local autocorrelation correction (Woolrich et al., 2001). The time-series GLM model included a double gamma HRF with temporal derivatives and motion parameters as nuisance regressors. The within-subject higher-level analysis was carried out using a fixed effects model by forcing the random effects variance to zero in FLAME (FMRIB's Local Analysis of Mixed Effects) (Beckmann et al., 2003; Woolrich et al., 2004). The z-scores were computed in each workflow separately for static and flicker conditions without spatial smoothing.

Conventional workflow: The functional runs were distortioncorrected with opposite phase-encoding images using a custom configuration file that was optimized for high-resolution data with TOPUP (Andersson et al., 2003) as implemented in FSL (Smith et al., 2004). The $T_{1}$ image from the MP2RAGE was then co-registered to the mean distortion-corrected functional image using SPM12. The statistical analysis in FSL FEAT for this workflow was carried out on the distortion-corrected functional datasets.

MI-EPI workflow: The anatomical $\mathrm{T}_{1}$ map computed from the MIEPI was co-registered to the mean functional image using SPM12 (Ashburner, 2007). Note that, in contrast to several high-resolution fMRI studies (e.g. Muckli et al. (2015); Olman et al. (2012); Polimeni et al. (2010a); Siero et al. (2015); Zimmermann et al. (2011)), we coregister the anatomical image to the functional data (e.g. Koopmans et al., 2011) to minimize smoothing and interpolation of the functional data.

Regions-of-interest:. The large values in the statistical maps are usually due to draining veins near the activated region (Gati et al., 1997; Haacke et al., 1994). These voxels were excluded from the analysis by thresholding the z-scores from the second-level FEAT analysis for each condition between $50 \%$ and $90 \%$ of the maximum z-score. This was done because the highest z-statistic values are very likely to arise from large signal amplitude in the pial vessels (Polimeni et al., 2010a) or voxels with high contribution of ascending veins and, thereby, the procedure improved the spatial specificity of the activation clusters by removing remaining vein-dominated voxels. An intersection of these thresholded z-maps from the flicker and static conditions was used to obtain clusters of voxels that were activated in both stimulus conditions. The two largest contiguous clusters were selected per subject yielding a total of ten ROIs in each analysis workflow. The statistical activation clusters were masked with the segmented GM ribbon from the anatomical data.

\section{Cortical depth analysis}

Cortical-depth definition:. The manually-corrected ROI segmentations from ITK-SNAP were exported as 3D surfaces for GM, WM and CSF. For each ROI, the three tissue surfaces were imported into MATLAB and were used to define the GM-CSF and WM-GM boundaries at five times the resolution of the acquired data. Ten depth-dependent surfaces were created including the GM-CSF and WM-GM boundaries using the equivolume principle (Leprince et al., 2015; Waehnert et al., 2014), thereby resulting in nine cortical depths in GM. Since a voxel in the acquired spatial resolution can lie across several cortical depths, the high-resolution equivolume depth surfaces generated were used to compute a three-dimensional partial-volume matrix of depth-dependence for every voxel's signal in the resampled ROI.

Cortical-depth sampling: The voxels from the functional data for all ROIs were resampled using nearest-neighbor interpolation to five times the acquired resolution and assigned to the different depths in a winner-takes-it-all approach using the depth-dependent partialvolume matrix i.e., voxels with the highest weighting to a specific depth in the depth-dependent partial-volume matrix are assigned to that particular depth and the signal time-courses were then averaged separately for each cortical depth.

Cortical-depth profile calculation: To examine the different components of the event-related time-courses, we computed the cortical-depth profiles by averaging the signal amplitudes between 6$18 \mathrm{~s}$ following the stimulus onset, i.e. PB signal, and 27-39 s, i.e. PSU, for each depth.

Cortical-depth profile normalization:. The two main vascular biases in GE fMRI, a) draining of $\mathrm{dHb}$ from lower to upper layers of the cortex via ascending veins and $b$ ) baseline $\mathrm{CBV}$ and relaxation parameters such as $\mathrm{T}_{2}{ }^{*}$ can be different at different depths. To examine if the depth-dependent neuronal specificity of the signal can be recovered from the data, we used a normalization approach. Normalization of depth-dependent profiles have been proposed previously in the context of resting-state fMRI (Guidi et al., 2016b; Polimeni et al., 2010c) using BOLD fluctuation power to probe connectivity across areas and with induced hypercapnia (Guidi et al., 2016a). These normalization approaches are motivated by the fact that the baseline parameters, such as venous $\mathrm{CBV}\left(\mathrm{V}_{0}\right)$, are assumed to be multiplicative for BOLD sensitivity and conforms to the single-vascular-compartment BOLD signal models (e.g. in Buxton et al. (2004), Davis et al. (1998)). Thus, under this assumption, the $\mathrm{V}_{0}$ bias can be accounted for by the division of depth-dependent signal amplitudes elicited for the two different stimuli or across time-points. We can describe this by formulating the BOLD signal change, $\Delta S$, as a product of two functions, 
$\Delta S=f\left(V_{0}, E_{0}, T_{2}^{*}, T E, \ldots\right) \bullet g(\Delta V, \Delta d H b)$

The term $f\left(V_{0}, E_{0}, T_{2}^{*}, T E, \ldots\right)$ is a function of the baseline physiological and physical parameters influencing the BOLD signal, i.e. baseline blood volume, $\mathrm{V}_{0}$, baseline oxygen extraction fraction, $\mathrm{E}_{0}$, baseline transverse relaxation time constant in GE measurements, $T_{2}^{*}$, and echotime, TE. The term $g(\Delta V, \Delta d H b)$ is a function of the change in physiological parameters due to activation, i.e. changes in $\mathrm{CBV}, \Delta \mathrm{V}$, and changes in $\mathrm{dHb}, \Delta \mathrm{dHb}$. In the context of depth-dependent studies, $g(\Delta V, \Delta d H b)$ consists approximately of two additive components stemming from local microvasculature and the draining ascending veins (Uludag et al., 2009). Both the Davis (Davis et al., 1998) and Buxton (Buxton et al., 2004) models can be formalized in this way. This is a one vascular-compartment model, which is well justified for the strong venous weighting of the GE fMRI signal (Uludag et al., 2009).

For a given cortical depth, $L=f\left(V_{0}, E_{0}, T_{2}^{*}, T E, \ldots\right)$ is constant.

Typically, for low- and high-resolution fMRI studies, two stimulus conditions are contrasted by a subtraction operation, resulting in:

$\Delta S_{\text {Flicker }}-\Delta S_{\text {Static }}=L \cdot\left(g_{\text {Flicker }}-g_{\text {Static }}\right)$

Note that the difference of the signal changes is still dependent on $L$. Since $L$ is not independently measured, the depth-dependent profile of the difference between conditions is weighted by the baseline physiology and relaxation parameters of the different layers.

For a given cortical depth, $L$ is constant for the two stimulus conditions and/or time points. Therefore, a division (rather than a subtraction) can eliminate the effect of $L$ and would be the more appropriate way to account for the baseline CBV:

$\frac{\Delta S_{\text {Flicker }}}{\Delta S_{\text {Static }}}=\left(\frac{g_{\text {Flicker }}}{g_{\text {Static }}}\right)$

Thus, calibrating the BOLD signal to another condition or time point (or baseline fluctuations (Guidi et al., 2016b; Polimeni et al., 2010c)) effectively removes the bias stemming from baseline physiological and physical parameters. For the case that neuronal profiles of two stimuli or time points are just scaled versions of each other (i.e. $g_{\text {Flicker }}=s c \cdot g_{\text {Static }}$ ) and assuming that the function $g$ is linear for a range of changes in $\mathrm{CBV}$ and $\mathrm{dHb}$ (which is justified for the signal range in the tissue).

That is, if two stimuli or time points evoke the same depthdependent neuronal profile with some scaling factor, $s c$, then the division approach yields a constant value for all layers. In other words, a deviation from a constant profile after division is an indicator of depth-dependent differences neuronal activity. For example, if the division approach yields a depth-dependent profile with constant values up to a specific layer, then the neuronal activities for all layers below this layer are similarly scaled when comparing both conditions/ time points. Therefore, the division approach can, on the one hand, be used to remove baseline parameter biases and on the other hand, be utilized as a tool to explore and characterize the cortical depth profiles by taking the ratio of the depth-dependent profiles between the two stimulus conditions (in this case, Flicker/Static) or between two different portions of the time-course (in this case, PSU/PB) (Kashyap et al., 2016; Siero et al., 2015). Note that this approach yields, in our study (see below), results comparable to animal studies: it has been shown (Yacoub et al., 2006; Zhao et al., 2007) that the PSU is better spatially localized in GM relative to the PB response.

Venous and CSF time-courses:. In low resolution fMRI studies, the most dominant source of the fMRI signal contrast stems from cortical surface veins located in the CSF. In order to study differences of the time-courses, the CSF and dominantly venous voxels were manually selected for all ROIs. The venous voxels were manually identified as those voxels, which have both low tSNR and low signal, thus, appearing dark (Menon et al., 1993; Ogawa et al., 1993) in the mean EPI image (see Supplementary Material Fig. 3). CSF voxels were selected from the tissue class segmentation of the $\mathrm{T}_{1}$ map for every ROI. Since venous voxels can be contaminated by CSF, the CSF voxels were selected as a subset of the CSF segmentation mask that does not overlap with the venous voxels. Event-related average time-courses were calculated from these venous and CSF voxels and compared with the mid-GM time-course from the depth-dependent analysis. To better visualize the differences, the three time-courses were normalized to the time-point corresponding to the end of the stimulus.

Relative ROI size analysis:. The size of the ROI and therefore, the number of voxels averaged is usually determined by the activation map in the region. Averaging depth-dependent signals across all the activated voxels in a relatively large ROI may affect the magnitude of the effect that one observes and this magnitude may differ between the two workflows. Note that, for studies probing higher-order cognitive processing, the activation ROI is usually much smaller in size than for the checkerboard stimuli utilized in this study. Therefore, the dependency of the size of the ROI on the magnitude of the differences between the two workflows was explored. The size of an ROI was calculated as the tangential distance along the GM for every slice at every cortical depth. The center or seed for the ROI was determined by the voxel showing the largest condition difference in GM after computing a contrast between flicker and static. Note that this does not imply largest differences between the workflows. This analysis was also repeated using randomly selected seed voxels in a ROI (see Supplementary Material Fig. 5).

Ten percent of the total length of the ROI ( $\sim 60$ voxels on average) was taken as the minimum ROI size to have enough number of voxels to obtain sufficient SNR. This ROI was grown tangentially in both directions by five percent on each side to obtain the next ROI, i.e. twenty percent total length. The GM ROI was thus grown tangentially in increments of ten percent till ninety percent of the total length was reached. In the case, wherein the edge of the ROI was reached in one direction, the ROI was grown by ten percent only in the other direction. Five percent on either end of the ROI was not included in this analysis to avoid any edge errors of the segmented ROI. The z-statistic maps were thresholded using $\mathrm{z}>2.3$ and a corrected cluster significance threshold of $\mathrm{p}=0.05$, which is the default option in FSL FEAT. This threshold was considerably more liberal than the one used as the activation mask in the depth-dependent analyses and yielded larger number of voxels per ROI, allowing us to grow the ROIs within a relatively larger patch of cortex while still within the activation mask. The cortical-depth profiles for the PB signal were calculated for each relative ROI size in both workflows. We calculated the magnitude of the depth-dependent signal differences between the workflows (MI-EPI minus Conventional) for the two stimulus conditions across different ROI sizes. The magnitude of the depth-dependent signal differences was then summed across depths and averaged across subjects to obtain the total difference in signal for every ROI size. Contrary to computing differences from the mean signal in the ROI, this approach provides a measure of the cortical depth-dependent differences.

\section{Results}

\section{Anatomical data}

\section{Quantitative $T_{1}$ comparison}

Four coronal slices from the Sa2RAGE-corrected MP2RAGE $T_{1}$ map (Fig. 2a) on a representative subject is shown next to the corresponding MI-EPI $\mathrm{T}_{1}$ map (Fig. $2 \mathrm{~b}$ ) to illustrate the quality of both anatomical datasets. The GM and WM quantitative $\mathrm{T}_{1}$ estimates in the occipital lobe for all subjects are listed in Table 1 . The average $\mathrm{T}_{1}$ estimate was calculated across four out of five subjects in whom 
Table 1

Quantitative $\mathrm{T}_{1}$ values of grey and white matter estimated with MP2RAGE and MI-EPI for all subjects. Note that $\mathrm{T}_{1}$ average was computed from Subjects 1-4 because the Sa2RAGE could not be acquired in Subject 5 .

\begin{tabular}{llllll}
\hline \multirow{2}{*}{ Subject } & \multicolumn{2}{l}{ Grey matter $\mathbf{T}_{\mathbf{1}}$ (in s) } & & \multicolumn{2}{l}{ White matter $\mathbf{T}_{\mathbf{1}}$ (in s) } \\
\cline { 2 - 3 } \cline { 6 - 6 } & MP2RAGE & IR-EPI & & MP2RAGE & \multicolumn{1}{l}{ IR-EPI } \\
\hline $\mathbf{1}$ & $1.79 \pm 0.27$ & $1.37 \pm 0.19$ & & $1.26 \pm 0.11$ & $0.94 \pm 0.13$ \\
$\mathbf{2}$ & $1.62 \pm 0.15$ & $1.56 \pm 0.25$ & & $1.19 \pm 0.09$ & $1.04 \pm 0.14$ \\
$\mathbf{3}$ & $1.64 \pm 0.25$ & $1.52 \pm 0.31$ & & $1.16 \pm 0.09$ & $0.93 \pm 0.19$ \\
$\mathbf{4}$ & $1.60 \pm 0.21$ & $1.39 \pm 0.24$ & & $1.12 \pm 0.07$ & $0.96 \pm 0.14$ \\
$\mathbf{5}$ & $1.90 \pm 0.19$ & $1.46 \pm 0.23$ & & $1.32 \pm 0.11$ & $0.98 \pm 0.15$ \\
Average & $\mathbf{1 . 6 6} \pm \mathbf{0 . 2 2}$ & $\mathbf{1 . 4 6} \pm \mathbf{0 . 2 5}$ & $\mathbf{1 . 1 8} \pm \mathbf{0 . 0 9}$ & $\mathbf{0 . 9 7} \pm \mathbf{0 . 1 5}$ \\
\hline
\end{tabular}

Sa2RAGE was acquired. The WM and $\mathrm{GM} \mathrm{T}_{1}$ estimates were shorter in MI-EPI when compared to the MP2RAGE (mean difference $0.215 \pm$ $0.078 \mathrm{~s}, \mathrm{p}=0.012$ and $0.202 \pm 0.157 \mathrm{~s}, \mathrm{p}=0.082$, respectively). All $\mathrm{p}$ values reported are adjusted for multiple comparisons using Bonferroni correction.

\section{Co-registration analysis}

In Fig. 3f, we show the probability density histogram for all voxels within the MI-EPI and the distorted-MP2RAGE GM masks in the medial ROI, indicating the mean EPI signal values of $196.30 \pm 10.59$ and $194.706 \pm 9.88$, respectively. For the lateral ROI (Fig. 3g), the mean EPI signals of all GM voxels in MI-EPI and distorted-MP2RAGE maps were $223.85 \pm 19.97$ and $221.55 \pm 22.64$, respectively. In both the medial and lateral ROIs, the mean signal from the non-overlapping voxels of the distorted-MP2RAGE was significantly lower compared to the mean GM EPI signal (mean difference for medial ROI: $26.42 \pm$ 5.32, $\mathrm{p}<0.001$, and for lateral ROI: $44.87 \pm 9.9, \mathrm{p}=0.001$ ). However, no significant difference between the mean values for the non-overlapping voxels was found for MI-EPI, indicating higher accuracy of the registration as compared to the MP2RAGE workflow. There was no statistically significant difference $(\mathrm{t}(8)=1.424, \mathrm{p}=0.1922)$ in the number of GM voxels across subjects between the MI-EPI $(16,090 \pm 844$ voxels) and the distorted-MP2RAGE (14,499 \pm 731 voxels $)$ ROIs used in this analysis.

\section{Functional data}

For illustrative purposes, the activation map from a single subject for the flicker condition from the MI-EPI workflow is shown in Fig. 4. The static condition also activated the same spatial subset of voxels as the flicker condition. An intersection of the thresholded z-statistic maps of flicker and static conditions in the ROIs was used for the subsequent depth-dependent analyses yielding an average of $160 \pm 19 \mathrm{~g}$ voxels (in the acquired resolution) per ROI. Robust activation clusters were detected in both ROIs and stimulus conditions for all subjects.

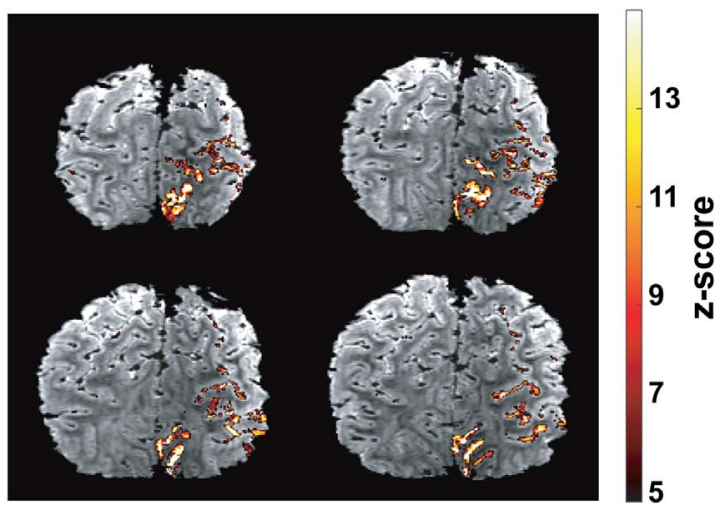

Fig. 4. Activation map of a single subject for the flickering stimulus overlaid on four slices of the mean EPI from the MI-EPI workflow.

a) Conventional Workflow

FLICKER

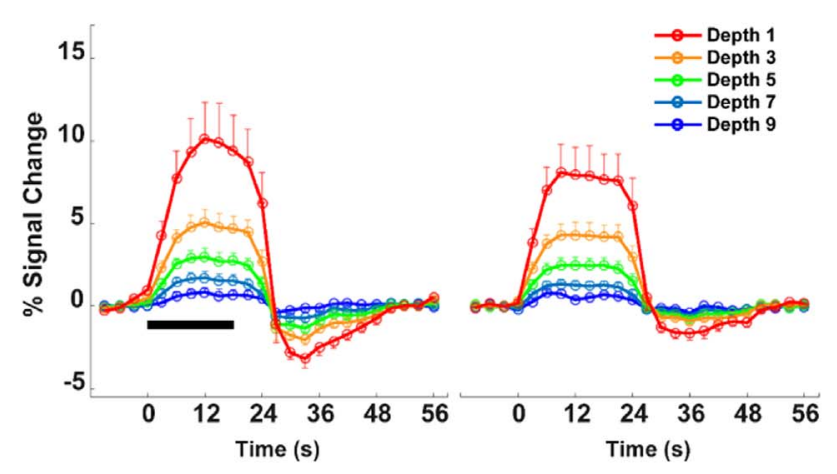

b) MI-EPI Workflow

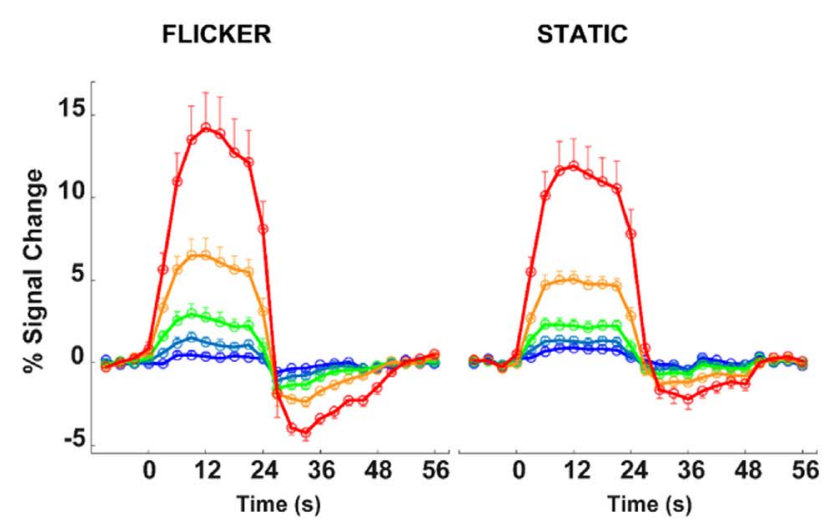

c) Depth profiles
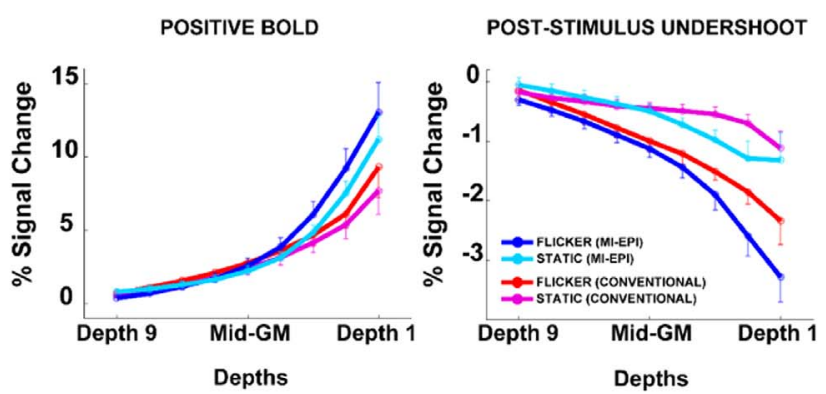

Fig. 5. Cortical depth-dependent time-courses (five out of nine GM depths shown) for the two stimulus conditions in the (a) Conventional and (b) MI-EPI workflows, respectively (horizontal black bar indicates stimulus duration). Cortical depth profiles for both conditions in two workflows for (c, left) PB and (c, right) PSU. Error bars indicate SEM. Depths 1 to 9 indicate GM depths between the CSF and the WM boundaries.

\section{Cortical depth-dependent analysis}

Event-related average time-courses computed for both stimulus conditions in the two workflows are shown in Fig. 5. The dynamic range of the signal amplitude of depth-dependent response timecourses is higher in the MI-EPI workflow (Fig. 5b) compared to the Conventional workflow (Fig. 5a) for both conditions. The flicker condition elicited a much stronger PSU than the static condition and is visible in both workflows. In both workflows, the cortical-depth profiles for the PB signal (Fig. 5c, left) show a signal increase towards the more superficial cortical-depths (towards the GM-CSF boundary) in both stimulus conditions, typical for GE fMRI acquisitions. The PB profile from the MI-EPI workflow differs significantly from the Conventional workflow in the two uppermost cortical-depths in both stimulus conditions (flicker: $\mathrm{F}_{(1,81)}=4.946, \mathrm{p}=0.0289$, mean difference 
at Depth $1=3.746 \% \mathrm{BOLD}, \mathrm{p}=0.0038$, mean difference at Depth $2=3.108 \%$ BOLD, $\mathrm{p}=0.0279$; static: $\mathrm{F}_{(1,81)}=7.458, \mathrm{p}=0.0077$, mean difference at Depth $1=3.494 \%$ BOLD, $p=0.0002$ ). In the case of the PSU for the flicker condition (Fig. 5c, right), a trend similar to the PB signal is observed with significant differences between workflows being at the most superficial depths $\left(\mathrm{F}_{(1,81)}=11.98, \mathrm{p}=0.0009\right.$, mean difference at Depth $1=-0.9436 \%$ BOLD, $p=0.0122)$. In the static condition, however, the differences in the PSU between the two workflows was most significant at the two cortical depths immediately below the most superficial one $\left(\mathrm{F}_{(1,81)}=5.205, \mathrm{p}=0.0251\right.$, mean difference at Depth $2=-0.5899 \%$ BOLD, $\mathrm{p}=0.0074$ ).

\section{GM, vein and CSF time-courses}

Fig. 6 shows the time-courses for mid-GM, venous and CSF voxels for both stimulus conditions. The differences in the BOLD times-topeak are not statistically significant $\left(\mathrm{F}_{(2,27)}=1.663, \mathrm{p}=0.208\right)$. We found a statistically significant difference in the magnitude of PSU between the three time-courses $\left(\mathrm{F}_{(1.02,6.117)}=10.29, \mathrm{p}=0.017\right)$, with a stronger undershoot in the mid-GM compared to the CSF (mean difference $=-0.2174 \pm 0.0851 \% \mathrm{BOLD}, \mathrm{p}=0.129$ ) and a significantly stronger mid-GM undershoot compared to the pial veins (mean difference $=-0.3504 \pm 0.1028 \% B O L D, p=0.043$ ). Similar time-course characteristics are present also during the static condition, albeit with a much smaller undershoot response.

\section{Relative ROI size analysis}

Fig. 7 illustrates the effect of increasing ROI size on the sum of the absolute depth-dependent differences of the PB signal between the MIEPI workflow and the Conventional workflow for the two stimulus conditions. In the results presented above, the average ROI size used was about $26 \%$ with respect to the scale shown in Fig. 7 ( $\sim 160$ voxels). A decrease in the total difference between the two workflows is observed with increasing ROI size for both stimulus conditions (Fig. 7). Despite averaging over the largest ROI size, there is still a 4.52\% BOLD (flicker) and 3.16\% BOLD (static) difference between the two workflows, which is about $13.57 \%$ and $10.68 \%$ (flicker and static, respectively) of the total depth-dependent PB signal in the ROI.

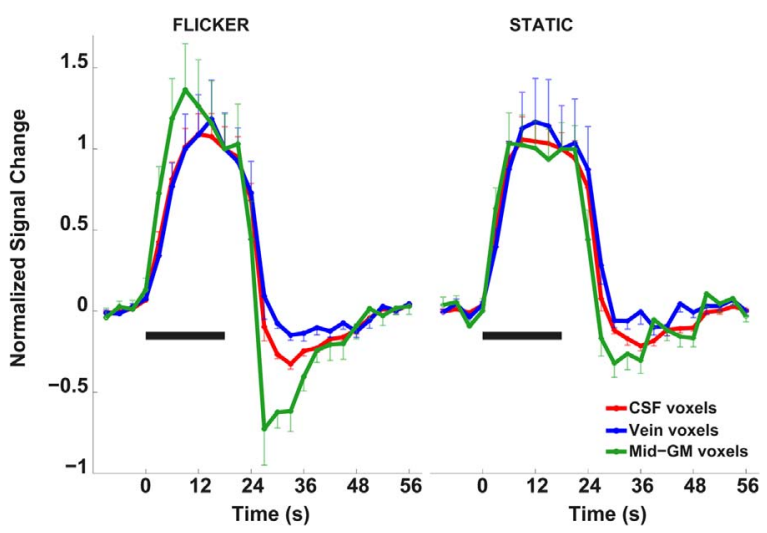

Fig. 6. Normalized event-related time-courses from voxels in the CSF and veins in the ROIs compared to the mid-GM time-course for the flicker (left) and static (right) conditions from the MI-EPI workflow. Error bars indicate SEM.

\section{Cortical-depth profile normalization}

Due to the fact that the strong differences can be driven by division of small BOLD signal changes in the deepest layers, we restricted our ANOVA analysis to compare seven of the nine cortical depths, not including the two GM depths near the WM boundary. In the first normalization (see Fig. 8a), the ratio of the PB depth profiles of the flicker relative to the static response in the Conventional workflow does not reveal any significant depth-specific modulation $\left(\mathrm{F}_{(6,63)}=0.208\right.$,

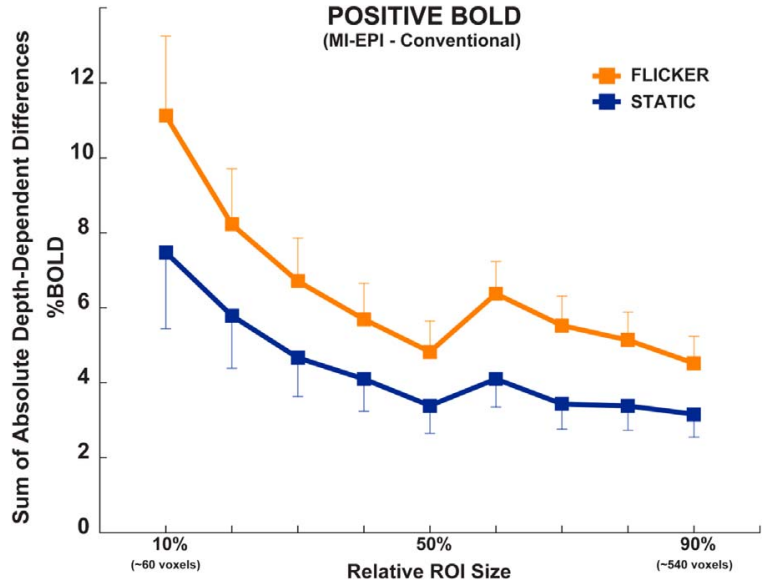

Fig. 7. The sum of absolute depth-dependent differences in BOLD signal between the workflows (MI-EPI minus Conventional) is shown with respect to increasing ROI size for the two stimulus conditions. Error bars indicate sum of squared errors.

$\mathrm{p}=0.97)$. However, in the MI-EPI workflow, we observe that the PB is proportionally larger in amplitude in the flicker compared to the static condition towards the pial surface and this effect reverses with increasing depth $\left(\mathrm{F}_{(6,63)}=2.154, \mathrm{p}=0.059\right)$. The ratio of depth profiles from different portions of the hemodynamic response, i.e. PSU to the PB (see Fig. 8b), shows no significant differences between MI-EPI and Conventional workflows for the static condition. However, the two workflows exhibit contrasting behavior for the flicker condition. In the MI-EPI workflow, the PSU increases proportionally to the PB signal with increasing depth. The Conventional workflow shows the opposite pattern.

\section{Discussion}

In the present study, we compared the implications of the data acquisition strategy on the subsequent processing and analysis of depth-dependent fMRI data (Fig. 1) and, additionally, presented an approach to reduce vascular bias in GE BOLD fMRI.

\section{Anatomical data}

The conventional data acquisition strategy involves differentlydistorted anatomical and functional data (in this case, MP2RAGE \& GE-EPI). We propose acquiring high-resolution anatomical data using MI-EPI instead. This approach has the advantage of being distortionmatched to the functional data. As a side result, the present study was well-placed to compare the $\mathrm{T}_{1}$ values over the occipital cortex (see Table 1). We observed shorter tissue $\mathrm{T}_{1}$ estimates with the MI-EPI than with the Sa2RAGE-corrected MP2RAGE. This could be, on the one hand, due to magnetization transfer (MT) effects from the fat saturation pulse used in MI-EPI, but not in the MP2RAGE (van Gelderen et al., 2016). In addition to MT-effects, imperfections in the excitation slice profiles could also cause partial saturation of the consecutive slice and, therefore, a potential bias in the $\mathrm{T}_{1}$ estimates. On the other hand, the estimation of $\mathrm{T}_{1}$ in MP2RAGE on just two inversion times may be prone to estimation errors. Clearly, more work has to be done to determine the most reliable approach for quantitative $\mathrm{T}_{1}$ estimation, which, however, is beyond the scope of the current study. Although quantitative $T_{1}$ determination may be a concern for myelin mapping studies, for the purposes of yielding sufficiently high tissue contrast for segmentation and cortical depth definition, the MI-EPI $\mathrm{T}_{1}$ map is comparable to the Sa2RAGE-corrected MP2RAGE $\mathrm{T}_{1}$ map (Fig. 2). Recently, MI-EPI has been shown to be of sufficient quality to perform automated whole-brain segmentation and surface-based analysis (Renvall et al., 2016). Lastly, the GRAPPA kernel used to reconstruct the multiple TI contrasts can introduce mild ghosting, therefore, the 
a)

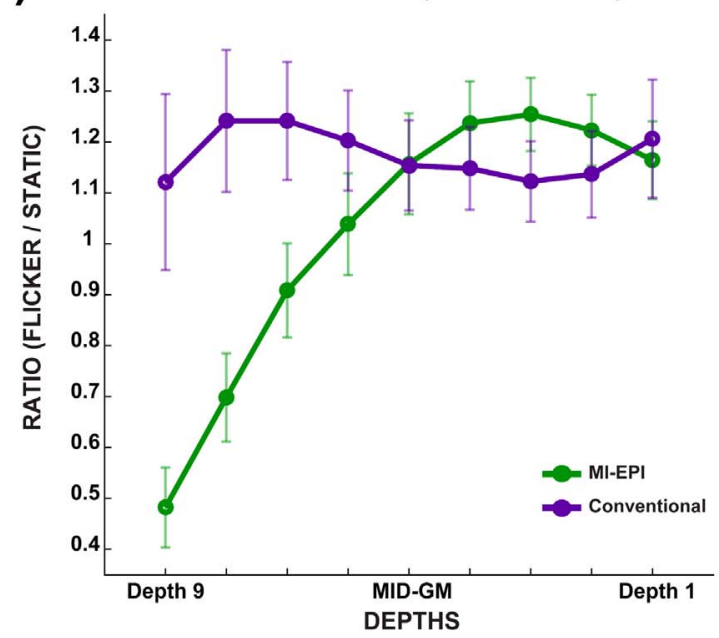

b)

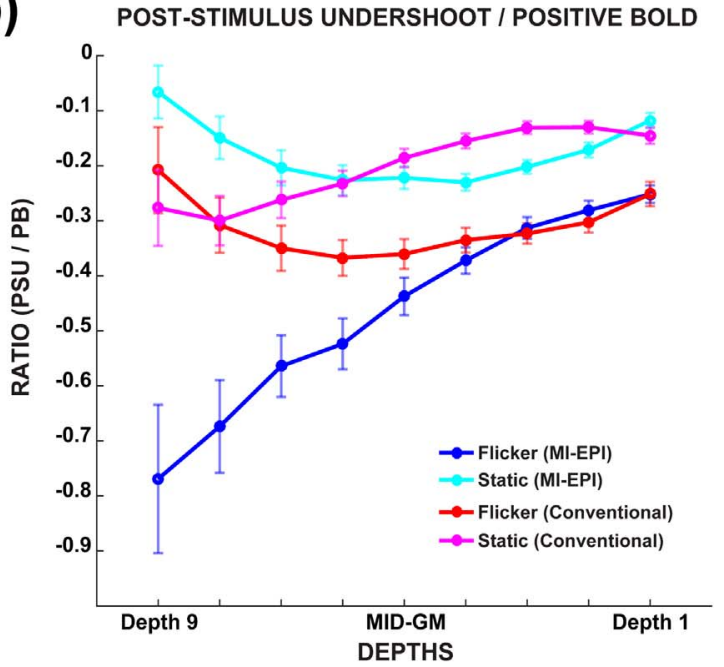

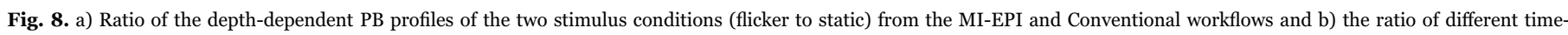
segments (PSU to $\mathrm{PB}$ ) for the two workflows and stimulus conditions.

reconstruction must be optimized (as in the present study) and each MI-EPI dataset must be carefully examined for the presence of such artefacts around the ROI.

\section{Co-registration quality}

The conventional acquisition strategy, utilized in low-resolution fMRI studies in particular, requires distortion-correcting the functional data using post-processing tools such as FSL TOPUP (Andersson et al., 2003), a preferred approach owing to some of the practical difficulties of the fieldmap-based distortion-correction (Andersson et al., 2001; Zeng and Constable, 2002). In distortion-correction approaches, the increased sensitivity to field inhomogeneities and sub-voxel subject motion limit the accuracy of estimating and correcting geometric distortions. Therefore, suboptimal distortion-correction can result in co-registration errors between the functional and anatomical data. We demonstrate the use of a histogram-based approach to quantify registration quality between anatomical and functional data. By distorting the MP2RAGE to the EPI space, we could quantitatively compare the registration of the mean EPI image with the MI-EPI $\mathrm{T}_{1}$ and the distorted-MP2RAGE. Note that we registered only partial FOV centered on the ROIs. Full FOV registration is not advised in highresolution fMRI studies as the cost function used by the registration algorithm takes irrelevant ROIs into account and thereby, increase registration errors on the relevant ROIs. The non-overlapping voxels were of interest in this analysis because they exclusively fall under the distorted-MP2RAGE or the MI-EPI GM mask (Fig. 3a-d).

The probability density histograms in the distorted-MP2RAGE shows that the non-overlapping voxels have significantly lower intensity than the mean GM signal. As we calculated the histograms from the same data (mean EPI in the native space), blurring due to sequence differences does not play a major role but rather the differences in the histograms indicate differences in the GM definition. Therefore, the shift of the peak (dashed lines) to the left (more WM contamination, lower signal) is an indicator of misregistration. In the MI-EPI workflow, the histogram of the non-overlapping and overlapping voxels from the GM mask are not significantly different indicating smaller degree of sampling CSF/WM signal and thus, demonstrates a high accuracy of registration. The effects of partial voluming with WM and CSF are region-dependent, the higher the underlying distortions are, the more likely it is that the GM fMRI signals are contaminated with those of WM and CSF, as illustrated in Fig. 3g.

\section{Functional data}

We successfully replicated an earlier study (Sadaghiani et al., 2009) at $7 \mathrm{~T}$ using similarly designed stimuli and show that it is consistent across both workflows. The goal of the stimulus was to evoke robust activation with sufficient SNR at this sub-millimeter spatial resolution and not to preferentially activate a certain visual area. The functional ROI clusters for all subjects were approximately located in V1 and V2, and the stimulus-evoked responses show robust depth-dependent behavior. However, as the goal of this study was to compare different processing and acquisition approaches for depth-dependent fMRI, the conclusions of this study are independent of the exact location of the activation.

The EPI readout was kept as short as possible to achieve our target resolution and coverage. Here, it was on the order of twice the grey matter's $\mathrm{T}_{2}{ }^{*}$ through the use of in-plane acceleration. It is important to keep in mind that longer readout trains may result in increased $\mathrm{T}_{2}{ }^{*}$ blurring (Goense and Logothetis, 2006; Huber et al., 2015b), which may affect the depth-dependent signal of interest. Both conventional and MI-EPI workflows resulted in robust activation maps for both stimulus conditions. The activation clusters were similarly spatially localized in both workflows. In contrast to most low- and highresolution fMRI studies, we co-register the anatomy to the mean functional image to minimize the post-processing and transformations done to the depth-dependent functional data. That is, this approach may already reduce smoothing associated with both the distortioncorrection and subsequent registration of the functional data.

\section{Cortical depth-dependent analysis}

The cortical depth profiles exhibit signal increases towards the uppermost depths, typical for GE-EPI shown previously (De Martino et al., 2013; Goense and Logothetis, 2006; Harel et al., 2006; Polimeni et al., 2010a; Uludag and Blinder, 2017; Zhao et al., 2006). A similar trend was observed for the depth profiles of the PSU (Puckett et al., 2016; Siero et al., 2015; Uludag and Blinder, 2017). The dynamic range of the depth-dependent responses is lower in the Conventional workflow compared to the MI-EPI workflow (Fig. 5a, b). In addition, the Conventional approach also results in a higher BOLD signal being observed in the lower cortical depths. The cortical depth profiles exhibit the differences between the two workflows in a more pronounced manner (Fig. 5c). For the PB response, significant differences between the workflows were found in the upper cortical depths (close to the CSF boundary) and, given that changes in neural activity induced via feedback are assumed to differ mostly in the upper laminae (Muckli et al., 
2015), choosing the right acquisition and analysis approaches are crucial for studying cognitive processes within the cortical laminae. Our findings indicate that post-processing steps, such as distortion-correction, can indeed affect the depth-dependent signal of interest. Registration errors can, in principle, add to the differences between the two workflows. As we did not observe a systematic shift of the registration between the GM masks of the two approaches (data not shown), registration errors cannot account for these observations. The lower dynamic range in the Conventional workflow, however, could be a consequence of the recalculation of the voxel intensities and smoothing during the distortion-correction step. Thus, both the reduced BOLD signal amplitude in the upper cortical depths and increased BOLD signal amplitude in the lower cortical depths for the Conventional approach are most likely due to the smoothing introduced by distortion-correction rather than registration errors. It is important to note that the responses were measured from a subset of voxels that was active for both stimuli. This ensured that there were no differences in the underlying vasculature amongst the voxels being averaged and compared across both conditions.

\section{Mid-GM, CSF and venous time-courses}

Given the high-resolution of our data, we were able to identify (see Supplementary material Fig. 3) and extract the event-related hemodynamic responses from veins and CSF voxels and compared them to the response in mid-GM (Fig. 6). The CSF voxels with significant functional activity in the present study most likely refers to voxels that contain smaller pial veins which are embedded in the CSF but are not resolvable given the spatial resolution utilized in this study. Although the PB response appears to be faster in the mid-GM, which would be consistent with the fact that the neuronally-driven hemodynamic response in GM is expected to exhibit faster dynamics than the venous response (Kennerley et al., 2010; Krings et al., 1999; Lee et al., 1995; Silva and Koretsky, 2002; Tian et al., 2010; Uludag and Blinder, 2017), we did not find a statistically significant difference in the times-to-peak between the time-courses. Interestingly, a stronger PSU is observed in the mid-GM (Huber et al., 2014) compared to the venous and CSF responses having little or no undershoot. Our finding argues that the PSU is better localized relative to the neuronal activity than the PB response (Sirotin et al., 2009; Uludag, 2010; Zhao et al., 2007). Importantly, the differences in the PSU for the two stimuli are best visible in the GM rather than on the surface. Therefore, the fMRI signal from the pial veins can smooth out the tissue fMRI response. Given that surface fMRI signals are larger in amplitude, our finding indicates that low-resolution fMRI studies may be less sensitive to true differences in neuronal activity, which are better represented by the tissue fMRI signals, thus, further emphasizing the need for ultra-high field high-resolution imaging. In the case of low-resolution GE fMRI studies, however, excluding the highest activated voxels representing signal from the pial veins may increase specificity for tissue specific signal that could more closely represent neuronal processing (Gati et al., 1997; Koopmans et al., 2010; Moon et al., 2007).

\section{Relative ROI size analysis}

Most depth-dependent fMRI studies rely on ROI-based analyses making the acquisition strategy and the subsequent data processing steps critical to investigate signal modulations across laminae. For large ROIs, small errors in registration and signal blurring that result in depth-dependent differences between the workflows can effectively cancel out because of averaging over a large cortical region. However, in most depth-dependent cognitive neuroscience studies, the investigated effect of interest occurs in small patches along the cortex. To this end, we investigated the depth-dependent differences between workflows by varying the sampled area, i.e. the ROI size (Fig. 7), for both stimulus conditions. Because most depth-dependent fMRI studies are interested in differences between tasks, the choice of the seed ROI was based on the voxels having the largest difference between stimulus conditions and not the highest activated voxels. The quantitative metric for the comparison was the absolute sum of differences in signal between the two workflows. We observed that the signal difference between the workflows is largest for the smallest ROI as this region has the highest signal change given our seed ROI criteria. By increasing the ROI size, i.e. averaging more voxels around the seed ROI, the depthdependent difference between workflows steadily decreases. Additionally, we bootstrapped the seed of our ROI, performed the same analysis and observed that the effect of ROI size is very similar (see Supplementary Material Fig. 5) to those seen in Fig. 7. This supports the argument that, in studies mapping large brain regions, the choice of workflow would not be critical. However, for depth-dependent fMRI studies, wherein small patches of cortex are typically of interest, the choice of analysis workflow has an impact on the depthdependent signal changes.

\section{Depth profile normalization}

The GE signal is largely biased by veins oriented parallel (pial) and, to a smaller extent, perpendicular (ascending) to the cortical surface (Gagnon et al., 2015; Gati et al., 1997; Menon et al., 1993; Uludag et al., 2009)... The underlying depth-dependent BOLD signal is biased mainly by a) draining of $\mathrm{dHb}$ from lower to upper layers of the cortex via ascending veins and b) depth-dependent differences in baseline CBV. Although the first bias is more pronounced with GE, the ascending veins can contribute to the degradation of depth-dependent specificity in SE as well. In this regard, initial steps towards modelbased removal of this bias have been carried out by Heinzle et al. (2016) and Markuerkiaga et al. (2016). Here, we propose to reduce the latter bias by dividing depth-dependent signals between conditions and/or time points and motivate this approach with theoretical considerations derived for one-vascular compartment models (Buxton et al., 2004; Davis et al., 1998), which is appropriate for GE fMRI (Uludag et al. (2009)).

By taking the ratio of $\mathrm{PB}$ profiles from the different stimulus conditions with the MI-EPI workflow, the modulation of depthdependent signal could be observed (Fig. 8a), in contrast to the Conventional workflow. In the second case, using the ratio of different portions of the time-course, we were able to reveal depth-dependent modulation of the PSU relative to the PB (Fig. 8b). The depth profile in the MI-EPI workflow for the flicker condition shows that the PSU is relatively stronger in the middle to the deeper cortical depths, consistent with results obtained on animal models (Yacoub et al., 2006; Zhao et al., 2007). However, this effect is not observed with the Conventional workflow. For the flicker condition using the MI-EPI workflow, we observe that the PB (Fig. 5c) is proportionally larger in the uppermost depths whereas the PSU (Fig. 8b) is relatively (but not absolutely) larger with increasing depth. The PSU and PB profiles represent two distinct temporal stages in the processing of the stimulus information and our data indicate that their ratio has different cortical depth dependence than the PB.

\section{Limitations and future considerations}

Our implementation of MI-EPI was time intensive ( $25 \mathrm{~min})$ because of the multiple inversions and repetitions acquired. In future studies, the MI-EPI can be made more efficient and optimized (Renvall et al., 2016) to significantly reduce the acquisition time. Alternative approaches to acquire sub-millimeter distortion-matched anatomy at $7 \mathrm{~T}$ include the T1-2-3DEPI (van der Zwaag et al., 2016) and $\mathrm{T}_{1}$-EPI (Huber et al., 2014). Despite the fact that the MI-EPI can help improve co-registration accuracy and provide anatomical contrast to define cortical depths, it is still an additional run to acquire. It would be ideal to use the endogenous anatomical contrast of the functional data itself. Recently, depth-dependent studies using VAscular Space Occupancy (VASO) to measure functional CBV changes (Huber et al., 2016a) show that it is possible to calculate anatomical contrast from the functional VASO data, thereby, circumventing the need to co-register datasets and 
distortion-correct altogether. However, this is currently not possible for standard BOLD fMRI studies and future work should explore whether adding RF pulses or analyzing additional features of fMRI data, such as the phase information or temporal standard deviation, might aid the segmentation of the GM ribbon without the need for an additional run. Until then, our workflow using the MI-EPI provides a good alternative to conventional approaches to acquire and analyze high-resolution depth-dependent fMRI at ultra-high fields. Additionally, it is possible that the GM thickness following distortion-correction in the functional data may be different than the MP2RAGE given the differences in phase-encode and readouts between the functional and anatomical datasets. This difference in GM thickness between the MP2RAGE and the functional (EPI) data can cause inaccurate cortical depth definitions even in the case of accurate co-registration and, therefore, incorrect sampling of the depth-dependent signal. While we did not test for this, it does not invalidate our conclusion that the MI-EPI workflow would not only overcome the first limitation of having to distortion-correct the data but also, given the quantitative nature of the anatomical map, more accurately represent the GM in the native EPI space.

Segmentation of GM, WM and CSF remains a challenge because most available data analysis packages are limited to automated wholebrain segmentation and need to be adapted for data with partial coverage. Although the GM ribbon can be reliably delineated due to the good anatomical contrast in both workflows, the sampled functional signal is still dependent on the processing strategy applied and the accuracy of co-registration. Additional analyses using GM segmentation from the distorted MP2RAGE and functional data in native EPI space demonstrate that the differences between the two workflows are mostly due to blurring induced by the distortion-correction in the Conventional workflow, rather than misregistration (see Supplementary Material Fig. 4), resulting in slightly higher BOLD amplitude in the deeper cortical depths and lower in the upper cortical depths in the Conventional approach compared to the MI-EPI approach. Each study should be carefully examined for confounds in the BOLD signal profiles resulting from both distortion-correction and registration related effects. Although we did not systematically investigate this effect, it is important to note that distortions in the phaseencoding direction can also introduce some blurring across layers and must be considered when selecting the acquisition orientation. It is important to carefully acquire the data such that the phase-encode blurring is preferentially within, rather than across the layers in an ROI that is activated by the stimulus (Huber et al., 2016b).

Despite the recent developments in high-resolution acquisition using different functional neuroimaging modalities such as CBV (Huber et al., 2015a) and CBF (Ivanov et al., 2016), GE BOLD fMRI remains the workhorse for high-resolution depth-dependent neuroimaging. In general for GE BOLD fMRI studies, care must be taken in interpreting depth-dependent signal changes in the upper layers in terms of neural processing (Yen et al., 2017). In this study, we propose a normalization approach based on existing BOLD signal models (Buxton et al., 2004; Davis et al., 1998) to uncover depth-dependent non-linearities of the GE BOLD signal. Note, however, the normalization approach still does not fully account for all the complexities of vascular biases in depth-dependent fMRI and, thus, we emphasize the need for a more comprehensive BOLD signal model to remove those biases in sub-millimeter depth-dependent fMRI studies. Thus, we propose the normalization approach as a tool to remove baseline parameter biases and detect non-linearities, most likely of neuronal origin, rather than accounting for all vascular biases in the depthdependent fMRI signals. Interpretations of normalized profiles need to be carefully considered because division by small numbers (for example, reduced GE BOLD SNR towards the WM boundary) may over-estimate the relative signal change in the deeper layers compared to the superficial layers.

Another important aspect for ROI-based analysis is that the BOLD signal is assumed to not vary tangentially within the analyzed segment at each cortical depth. This assumption of invariance is why voxels are averaged throughout the ROI in a depth-dependent manner. However, future studies must consider studying the signal variation along the cortical sheet for their significance in cortical processing and initial attempts, such as the cortical laminagram (Havlicek and Uludag, 2016), show promise. Future studies can consider using a spatial GLM to sample the depth-dependent fMRI signal (Havlicek and Uludag, 2016; Kashyap et al., 2016; Polimeni et al., 2010b; van Mourik et al., 2015) wherein the signal within each voxel is modeled as a linear combination of signal contributions from different cortical depths. The spatial GLM approach has been shown to have a higher specificity compared to the commonly used resampling approach (Havlicek and Uludag, 2016). However, more work needs to be done in order to improve and validate the spatial GLM approach for highresolution depth-dependent fMRI.

\section{Conclusions}

In this study, we presented and evaluated a processing workflow for depth-dependent analysis of submillimeter spatial resolution GE BOLD fMRI data at $7 \mathrm{~T}$ using a distortion-matched $\mathrm{T}_{1}$ map from MI-EPI. We show that minimally processed fMRI data can be analyzed in its native EPI space and this is facilitated by acquiring distortion-matched anatomical contrast using MI-EPI. We demonstrate that the distortion-correction methods can reduce the specificity of the depthdependent fMRI signal. We confirm that the PSU is spatially better localized in GM relative to the positive BOLD, and is proportionally larger in the GM than the veins. In addition, we provide a theoretical basis and demonstrate that the depth-dependent modulation of the neuronal signal can be, at least partially, recovered from GE BOLD signal by dividing the depth profiles from the different conditions or portions of the time-course. In conclusion, the choices made for acquisition strategy, processing and analysis in high-resolution depth-dependent fMRI data can significantly impact the observed depth-dependent effects and the possible outcome of studies. A comprehensive modeling, acquisition and analysis approach is needed to deconvolve the depth-dependent neuronal activation from BOLD fMRI signals coupled to each other at various cortical depths.

\section{Acknowledgements}

The research was supported by the Netherlands Organization for Scientific Research (NWO) VIDI 452-11-002 to Kâmil Uludağ. We would like to thank Laurentius Huber for the implementation of the trFOCI inversion pulse in the MI-EPI sequence and Jose P. Marques for providing us with the quantitative $\mathrm{T}_{1}$ correction scripts.

\section{Appendix A. Supporting information}

Supplementary data associated with this article can be found in the online version at doi:10.1016/j.neuroimage.2017.05.022.

\section{References}

Andersson, J.L., Hutton, C., Ashburner, J., Turner, R., Friston, K., 2001. Modeling geometric deformations in EPI time series. NeuroImage 13, 903-919.

Andersson, J.L., Skare, S., Ashburner, J., 2003. How to correct susceptibility distortions in spin-echo echo-planar images: application to diffusion tensor imaging. NeuroImage 20, 870-888.

Ashburner, J., 2007. A fast diffeomorphic image registration algorithm. NeuroImage 38, 95-113.

Ashburner, J., Friston, K.J., 2005. Unified segmentation. NeuroImage 26, 839-851.

Beckmann, C.F., Jenkinson, M., Smith, S.M., 2003. General multilevel linear modeling for group analysis in FMRI. NeuroImage 20, 1052-1063.

Brodmann, K., 1909. Vergleichende Lokalisationslehre der Grosshirnrinde in ihren Prinzipien dargestellt auf Grund des Zellenbaues. Barth.

Buxton, R.B., Uludag, K., Dubowitz, D.J., Liu, T.T., 2004. Modeling the hemodynamic response to brain activation. NeuroImage 23 (Suppl 1), S220-S233. 
Clare, S., Jezzard, P., 2001. Rapid T-1 mapping using multislice echo planar imaging. Magn. Reson. Med. 45, 630-634.

Davis, T.L., Kwong, K.K., Weisskoff, R.M., Rosen, B.R., 1998. Calibrated functional MRI: mapping the dynamics of oxidative metabolism. Proc. Natl. Acad. Sci. USA 95, 1834-1839.

De Martino, F., Moerel, M., Ugurbil, K., Goebel, R., Yacoub, E., Formisano, E., 2015. Frequency preference and attention effects across cortical depths in the human primary auditory cortex. Proc. Natl. Acad. Sci. USA 112, 16036-16041.

De Martino, F., Zimmermann, J., Muckli, L., Ugurbil, K., Yacoub, E., Goebel, R., 2013 Cortical Depth Dependent Functional Responses in Humans at 7T: improved Specificity with 3D GRASE. PLoS ONE 8, 30-32.

Douglas, R.J., Martin, K.A., 2004. Neuronal circuits of the neocortex. Annu. Rev. Neurosci. 27, 419-451.

Eggenschwiler, F., Kober, T., Magill, A.W., Gruetter, R., Marques, J.P., 2012. SA2RAGE: a new sequence for fast B1+-mapping. Magn. Reson. Med. 67, 1609-1619.

Fracasso, A., Luijten, P.R., Dumoulin, S.O., Petridou, N., 2017. Laminar imaging of positive and negative BOLD in human visual cortex at 7T. NeuroImage.

Fritz, L., Mulders, J., Breman, H., Peters, J., Bastiani, M., Roebroeck, A., Andersson, J.L., Ashburner, J., Weiskopf, N., Goebel, R., 2014. . Comparison of EPI distortion correction methods at 3T and 7T. In: Proceedings of Organisation for Human Brain Mapping, Hamburg, Germany.

Gagnon, L., Sakadzic, S., Lesage, F., Musacchia, J.J., Lefebvre, J., Fang, Q., Yucel, M.A., Evans, K.C., Mandeville, E.T., Cohen-Adad, J., Polimeni, J.R., Yaseen, M.A., Lo, E.H., Greve, D.N., Buxton, R.B., Dale, A.M., Devor, A., Boas, D.A., 2015. Quantifying the microvascular origin of BOLD-fMRI from first principles with two-photon microscopy and an oxygen-sensitive nanoprobe. J. Neurosci. 35, 3663-3675.

Gati, J.S., Menon, R.S., Ugurbil, K., Rutt, B.K., 1997. Experimental determination of the BOLD field strength dependence in vessels and tissue. Magn. Reson. Med. 38, 296-302.

Goense, J.B., Logothetis, N.K., 2006. Laminar specificity in monkey V1 using highresolution SE-fMRI. Magn. Reson. Imaging 24, 381-392.

Gowland, P., Mansfield, P., 1993. Accurate measurement of T1 in vivo in less than $3 \mathrm{~s}$ using echo-planar imaging. Magn. Reson. Med. 30, 351-354.

Griswold, M.A., Jakob, P.M., Heidemann, R.M., Nittka, M., Jellus, V., Wang, J., Kiefer, B., Haase, A., 2002. Generalized autocalibrating partially parallel acquisitions (GRAPPA). Magn. Reson. Med. 47, 1202-1210.

Guidi, M., Huber, L., Lampe, L., Gauthier, C.J., Moller, H.E., 2016a. Lamina-dependent calibrated BOLD response in human primary motor cortex. NeuroImage 141, $250-261$.

Guidi, M., Huber, L., Lampe, L., Moller, H.E., 2016b. Cortical laminar resting-state fluctuations scale with hypercapnic response. In: Proceedings of the 24th Annual Meeting of ISMRM, Singapore, Singapore.

Haacke, E.M., Hopkins, A., Lai, S., Buckley, P., Friedman, L., Meltzer, H., Hedera, P., Friedland, R., Klein, S., Thompson, L., et al., 1994. 2D and 3D high resolution gradient echo functional imaging of the brain: venous contributions to signal in motor cortex studies. NMR Biomed. 7, 54-62.

Haast, R.A.M., Ivanov, D., Formisano, E., Uludağ, K., 2016. Reprod. Reliab. Quant. Weight. T1 T2* Mapp. Myelin-Based Cortical Parcel. 7 T. Front. Neuroanat., 10.

Harel, N., Lin, J., Moeller, S., Ugurbil, K., Yacoub, E., 2006. Combined imaginghistological study of cortical laminar specificity of fMRI signals. NeuroImage 29, 879-887.

Havlicek, M., Uludag, K., 2016. Towards an optimal analysis of laminar-resolved fMRI. Proceedings of Organisation for Human Brain Mapping, Geneva, Switzerland.

Heinzle, J., Koopmans, P.J., den Ouden, H.E., Raman, S., Stephan, K.E., 2016. A hemodynamic model for layered BOLD signals. NeuroImage 125, 556-570.

Hoogenraad, F.G., Hofman, M.B., Pouwels, P.J., Reichenbach, J.R., Rombouts, S.A., Haacke, E.M., 1999. Sub-millimeter fMRI at 1.5 T: correlation of high resolution with low resolution measurements. J. Magn. Reson. Imaging 9, 475-482.

Huber, L., Goense, J., Kennerley, A.J., Ivanov, D., Krieger, S.N., Lepsien, J., Trampel, R., Turner, R., Moller, H.E., 2014. Investigation of the neurovascular coupling in positive and negative BOLD responses in human brain at 7 T. NeuroImage 97, $349-362$.

Huber, L., Goense, J., Kennerley, A.J., Trampel, R., Guidi, M., Reimer, E., Ivanov, D., Neef, N., Gauthier, C.J., Turner, R., Moller, H.E., 2015a. Cortical lamina-dependent blood volume changes in human brain at 7 T. NeuroImage 107, 23-33.

Huber, L., Guidi, M., Goense, J., Mildner, T., Trampel, R., Schulz, J., Eichner, C., Turner, R., Moller, H.E., 2015b. The magnitude point spread function is an inadequate measure of T2*-blurring in EPI. In: Proceedings of the 23rd Annual Meeting of ISMRM, Toronto, Canada.

Huber, L., Ivanov, D., Guidi, M., Turner, R., Uludag, K., Moller, H.E., Poser, B.A., 2016a. Functional cerebral blood volume mapping with simultaneous multi-slice acquisition. NeuroImage 125, 1159-1168.

Huber, L., Ivanov, D., Handwerker, D.A., Marrett, S., Guidi, M., Uludag, K., Bandettini, P.A., Poser, B.A., 2016b. Techniques for blood volume fMRI with VASO: from lowresolution mapping towards sub-millimeter layer-dependent applications. NeuroImage.

Hurley, A.C., Al-Radaideh, A., Bai, L., Aickelin, U., Coxon, R., Glover, P., Gowland, P.A., 2010. Tailored RF pulse for magnetization inversion at ultrahigh field. Magn. Reson. Med. 63, 51-58.

Hutton, C., Bork, A., Josephs, O., Deichmann, R., Ashburner, J., Turner, R., 2002. Image distortion correction in fMRI: a quantitative evaluation. NeuroImage 16, 217-240.

Ivanov, D., Poser, B.A., Huber, L., Pfeuffer, J., Uludag, K., 2016. Optimization of simultaneous multislice EPI for concurrent functional perfusion and BOLD signal measurements at 7T. Magn. Reson Med.

Jezzard, P., 2012. Correction of geometric distortion in fMRI data. NeuroImage 62, 648-651.
Jezzard, P., Balaban, R.S., 1995. Correction for geometric distortion in echo planar images from B0 field variations. Magn. Reson. Med. 34, 65-73.

Jezzard, P., Clare, S., 1999. Sources of distortion in functional MRI data. Hum. Brain Mapp. 8, 80-85.

Kashyap, S., Ivanov, D., Havlicek, M., Poser, B.A., Uludag, K., 2016. . High-resolution T1mapping using inversion-recovery EPI and application to cortical depth-dependent fMRI at 7 T. In: Proceedings of the 24th Annual Meeting of ISMRM, Singapore, Singapore.

Kemper, V.G., De Martino, F., Vu, A.T., Poser, B.A., Feinberg, D.A., Goebel, R., Yacoub, E., 2015. Sub-millimeter T2 weighted fMRI at 7 T: comparison of 3D-GRASE and 2D SE-EPI. Front Neurosci. 9, 163.

Kennerley, A.J., Mayhew, J.E., Redgrave, P., Berwick, J., 2010. Vascular origins of BOLD and CBV fMRI signals: statistical mapping and histological sections compared. Open Neuroimag J 4, 1-8.

Kim, D.S., Duong, T.Q., Kim, S.G., 2000. High-resolution mapping of iso-orientation columns by fMRI. Nat. Neurosci. 3, 164-169.

Kok, P., Bains, L.J., van Mourik, T., Norris, D.G., de Lange, F.P., 2016. Selective activation of the deep layers of the human primary visual cortex by top-down feedback. Curr. Biol. 26, 371-376.

Koopmans, P.J., Barth, M., Norris, D.G., 2010. Layer-specific BOLD activation in human V1. Hum. Brain Mapp. 31, 1297-1304.

Koopmans, P.J., Barth, M., Orzada, S., Norris, D.G., 2011. Multi-echo fMRI of the cortical laminae in humans at 7T. NeuroImage 56, 1276-1285.

Krings, T., Erberich, S.G., Roessler, F., Reul, J., Thron, A., 1999. MR blood oxygenation level-dependent signal differences in parenchymal and large draining vessels: implications for functional MR imaging. AJNR Am. J Neuroradiol. 20, 1907-1914.

Kwong, K.K., Belliveau, J.W., Chesler, D.A., Goldberg, I.E., Weisskoff, R.M., Poncelet, B.P., Kennedy, D.N., Hoppel, B.E., Cohen, M.S., Turner, R., 1992. Dynamic magnetic resonance imaging of human brain activity during primary sensory stimulation. Proc. Natl. Acad. Sci. USA 89, 5675-5679.

Lee, A.T., Glover, G.H., Meyer, C.H., 1995. Discrimination of large venous vessels in time-course spiral blood-oxygen-level-dependent magnetic-resonance functional neuroimaging. Magn. Reson. Med. 33, 745-754.

Leprince, Y., Poupon, F., Delzescaux, T., Hasboun, D., Poupon, C., Rivière, D., 2015. Combined Laplacian-equivolumic model for studying cortical lamination with ultra high field MRI (7 T). 2015 IEEE 12th Int. Symp. Biomed. Imaging (ISBI), 580-583.

Logothetis, N.K., 2008. What we can do and what we cannot do with fMRI. Nature 453, 869-878.

Logothetis, N.K., Merkle, H., Augath, M., Trinath, T., Ugurbil, K., 2002. Ultra highresolution fMRI in monkeys with implanted RF coils. Neuron 35, 227-242.

Mandeville, J.B., Marota, J.J., Kosofsky, B.E., Keltner, J.R., Weissleder, R., Rosen, B.R., Weisskoff, R.M., 1998. Dynamic functional imaging of relative cerebral blood volume during rat forepaw stimulation. Magn. Reson. Med. 39, 615-624.

Mansfield, P., Guilfoyle, D.N., Ordidge, R.J., Coupland, R.E., 1986. Measurement of T1 by echo-planar imaging and the construction of computer-generated images. Phys. Med Biol. 31, 113-124.

Markuerkiaga, I., Barth, M., Norris, D.G., 2016. A cortical vascular model for examining the specificity of the laminar BOLD signal. NeuroImage 132, 491-498.

Marques, J.P., Gruetter, R., 2013. New developments and applications of the MP2RAGE sequence-focusing the contrast and high spatial resolution R1 mapping. PLoS ONE 8, (e69294-e69294).

Marques, J.P., Kober, T., Krueger, G., van der Zwaag, W., Van de Moortele, P.F., Gruetter, R., 2010. MP2RAGE, a self bias-field corrected sequence for improved segmentation and T1-mapping at high field. NeuroImage 49, 1271-1281.

Mathiesen, C., Caesar, K., Akgören, N., Lauritzen, M., 1998. Modification of activitydependent increases of cerebral blood flow by excitatory synaptic activity and spikes in rat cerebellar cortex. J. Physiol. 512, 555-566.

Menon, R.S., Ogawa, S., Tank, D.W., Ugurbil, K., 1993. 4 T gradient recalled echo characteristics of photic stimulation-induced signal changes in the human primary visual cortex. Magn. Reson Med 30, 380-386.

Moon, C.H., Fukuda, M., Park, S.H., Kim, S.G., 2007. Neural interpretation of blood oxygenation level-dependent fMRI maps at submillimeter columnar resolution. J Neurosci. 27, 6892-6902.

Muckli, L., De Martino, F., Vizioli, L., Petro, L.S., Smith, F.W., Ugurbil, K., Goebel, R., Yacoub, E., 2015. Contextual Feedback to Superficial Layers of V1. Curr. Biol. 25, 2690-2695.

Mugler, J.P., Brookeman, J.R., 1990. Three-dimensional magnetization-prepared rapid gradient-echo imaging (3D MP RAGE). Magn. Reson Med 15, 152-157.

Mullinger, K.J., Mayhew, S.D., Bagshaw, A.P., Bowtell, R., Francis, S.T., 2013. Poststimulus undershoots in cerebral blood flow and BOLD fMRI responses are modulated by poststimulus neuronal activity. Proc. Natl. Acad. Sci. U S A 110, 13636-13641.

Munger, P., Crelier, G.R., Peters, T.M., Pike, G.B., 2000. An inverse problem approach to the correction of distortion in EPI images. IEEE Trans. Med Imaging 19, 681-689.

Nasr, S., Polimeni, J.R., Tootell, R.B., 2016. Interdigitated Color- and Disparity-Selective Columns within Human Visual Cortical Areas V2 and V3. J Neurosci. 36, 1841-1857.

Norris, D.G., 2012. Spin-echo fMRI: the poor relation? NeuroImage 62, 1109-1115.

Ogawa, S., Lee, T.M., Kay, A.R., Tank, D.W., 1990a. Brain magnetic resonance imaging with contrast dependent on blood oxygenation. Proc. Natl. Acad. Sci. U S A 87, 9868-9872.

Ogawa, S., Lee, T.M., Nayak, A.S., Glynn, P., 1990b. Oxygenation-sensitive contrast in magnetic resonance image of rodent brain at high magnetic fields. Magn. Reson. Med. 14, 68-78.

Ogawa, S., Menon, R.S., Tank, D.W., Kim, S.G., Merkle, H., Ellermann, J.M., Ugurbil, K., 1993. Functional brain mapping by blood oxygenation level-dependent contrast 
magnetic resonance imaging. A comparison of signal characteristics with a biophysical model. Biophys. J. 64, 803-812.

Olman, C.A., Harel, N., Feinberg, D.A., He, S., Zhang, P., Ugurbil, K., Yacoub, E., 2012. Layer-Specific fMRI Reflects Different Neuronal Computations at Different Depths in Human V1. PLoS ONE 7, (e32536-e32536).

Ordidge, R.J., Gibbs, P., Chapman, B., Stehling, M.K., Mansfield, P., 1990. High-speed multislice T1 mapping using inversion-recovery echo-planar imaging. Magn. Reson Med 16, 238-245.

Peirce, J.W., 2007. PsychoPy-Psychophysics software in Python. J Neurosci. Methods $162,8-13$.

Pfeuffer, J., van de Moortele, P.F., Yacoub, E., Shmuel, A., Adriany, G., Andersen, P., Merkle, H., Garwood, M., Ugurbil, K., Hu, X.P., 2002. Zoomed functional imaging in the human brain at $7 \mathrm{~T}$ with simultaneous high spatial and high temporal resolution. NeuroImage 17, 272-286.

Polimeni, J.R., Bhat, H., Witzel, T., Benner, T., Feiweier, T., Inati, S.J., Renvall, V., Heberlein, K., Wald, L.L., 2016. Reducing sensitivity losses due to respiration and motion in accelerated echo planar imaging by reordering the autocalibration data acquisition. Magn. Reson Med 75, 665-679.

Polimeni, J.R., Fischl, B., Greve, D.N., Wald, L.L., 2010a. Laminar analysis of 7T BOLD using an imposed spatial activation pattern in human V1. NeuroImage 52, 1334-1346.

Polimeni, J.R., Greve, D.N., Fischl, B., Wald, L.L., 2010b. Depth-resolved laminar analysis of resting-state fluctuation amplitude in high-resolution 7T fMRI. Proceedings of the Joint Meeting of ISMRM-ESMRMB, Stockholm, Sweden.

Polimeni, J.R., Witzel, T., Fischl, B., Greve, D.N., Wald, L.L., 2010c. Identifying common-source driven correlations in resting-state fMRI via laminar-specific analysis in the human visual cortex. Proceedings of the Joint Meeting of ISMRMESMRMB, Stockholm, Sweden.

Poser, B.A., Koopmans, P.J., Witzel, T., Wald, L.L., Barth, M., 2010. Three dimensional echo-planar imaging at 7 T. NeuroImage 51, 261-266.

Poser, Benedikt A., Setsompop, Kawin, 2017. Pulse sequences and parallel imaging for high spatiotemporal resolution MRI at ultra-high field. NeuroImage.

Pruessmann, K.P., Weiger, M., Scheidegger, M.B., Boesiger, P., 1999. SENSE: sensitivity encoding for fast MRI. Magn. Reson Med 42, 952-962.

Puckett, A.M., Aquino, K.M., Robinson, P.A., Breakspear, M., Schira, M.M., 2016. The spatiotemporal hemodynamic response function for depth-dependent functional imaging of human cortex. NeuroImage 139, 240-248.

Renvall, V., Witzel, T., Wald, L.L., Polimeni, J.R., 2016. Automatic cortical surface reconstruction of high-resolution T1 echo planar imaging data. NeuroImage 134, $338-354$

Ress, D., Glover, G.H., Liu, J., Wandell, B., 2007. Laminar profiles of functional activity in the human brain. NeuroImage 34, 74-84.

Sadaghiani, S., Ugurbil, K., Uludag, K., 2009. Neural activity-induced modulation of BOLD poststimulus undershoot independent of the positive signal. Magn. Reson Imaging 27, 1030-1038.

Siero, J.C., Hendrikse, J., Hoogduin, H., Petridou, N., Luijten, P., Donahue, M.J., 2015. Cortical depth dependence of the BOLD initial dip and poststimulus undershoot in human visual cortex at 7 T. Magn. Reson Med 73, 2283-2295.

Silva, A.C., Koretsky, A.P., 2002. Laminar specificity of functional MRI onset times during somatosensory stimulation in rat. Proc. Natl. Acad. Sci. U S A 99 15182-15187.

Sirotin, Y.B., Hillman, E.M., Bordier, C., Das, A., 2009. Spatiotemporal precision and hemodynamic mechanism of optical point spreads in alert primates. Proc. Natl. Acad. Sci. U S A 106, 18390-18395.

Smith, S.M., Jenkinson, M., Woolrich, M.W., Beckmann, C.F., Behrens, T.E., JohansenBerg, H., Bannister, P.R., De Luca, M., Drobnjak, I., Flitney, D.E., Niazy, R.K., Saunders, J., Vickers, J., Zhang, Y., De Stefano, N., Brady, J.M., Matthews, P.M., 2004. Advances in functional and structural MR image analysis and implementation as FSL. NeuroImage 23 (Suppl 1), S208-S219.

Stehling, M.K., Ordidge, R.J., Coxon, R., Mansfield, P., 1990. Inversion-recovery echoplanar imaging (IR-EPI) at 0.5 T. Magn. Reson Med 13, 514-517.

Stehling, M.K., Turner, R., Mansfield, P., 1991. Echo-planar imaging: magnetic resonance imaging in a fraction of a second. Science 254, 43-50.

Stikov, N., Boudreau, M., Levesque, I.R., Tardif, C.L., Barral, J.K., Pike, G.B., 2015. On the accuracy of T1 mapping: searching for common ground. Magn. Reson Med 73, $514-522$.

Tian, P., Teng, I.C., May, L.D., Kurz, R., Lu, K., Scadeng, M., Hillman, E.M., De Crespigny, A.J., D'Arceuil, H.E., Mandeville, J.B., Marota, J.J., Rosen, B.R., Liu, T.T.,
Boas, D.A., Buxton, R.B., Dale, A.M., Devor, A., 2010. Cortical depth-specific microvascular dilation underlies laminar differences in blood oxygenation leveldependent functional MRI signal. Proc. Natl. Acad. Sci. U S A 107, 15246-15251.

Ugurbil, K., Adriany, G., Andersen, P., Chen, W., Garwood, M., Gruetter, R., Henry, P.G., Kim, S.G., Lieu, H., Tkac, I., Vaughan, T., Van De Moortele, P.F., Yacoub, E., Zhu, X.H., 2003a. Ultrahigh field magnetic resonance imaging and spectroscopy. Magn. Reson Imaging 21, 1263-1281.

Ugurbil, K., Toth, L., Kim, D.S., 2003b. How accurate is magnetic resonance imaging of brain function? Trends Neurosci. 26, 108-114.

Uludag, K., 2010. To dip or not to dip: reconciling optical imaging and fMRI data. Proc. Natl. Acad. Sci. U S A, 107, (E23; author reply E24-E23; author reply E24).

Uludag, K., Blinder, P., 2017. Linking brain vascular physiology to hemodynamic response in ultra-high field MRI. NeuroImage.

Uludag, K., Muller-Bierl, B., Ugurbil, K., 2009. An integrative model for neuronal activity-induced signal changes for gradient and spin echo functional imaging. NeuroImage 48, 150-165.

Uludağ, K., Uğurbil, K., Berliner, L., 2015. FMRI: from Nuclear Spins to Brain Functions.. Springer.

van der Zwaag, W., Buur, P., Versluis, M., Marques, J.P., 2016. . Distortion-matched T1maps and bias-corrected T1w-images as anatomical reference for submillimeterresolution fMRI., In: Proceedings of the 24th Annual Meeting of ISMRM, Singapore, Singapore.

van Gelderen, P., Jiang, X., Duyn, J.H., 2016. Effects of magnetization transfer on T1 contrast in human brain white matter. NeuroImage 128, 85-95.

van Mourik, T., van der Eerden, J.P.J.M., Norris, D., 2015. . Laminar time course extraction over extended cortical areas. In: Proceedings of the 23rd Annual Meeting of ISMRM, Toronto, Canada.

Vaughan, J.T., Garwood, M., Collins, C.M., Liu, W., DelaBarre, L., Adriany, G., Andersen, P., Merkle, H., Goebel, R., Smith, M.B., Ugurbil, K., 2001. 7T vs. 4T: rf power, homogeneity, and signal-to-noise comparison in head images. Magn. Reson Med 46, 24-30.

Vaughan, J.T., Griffiths, J.R., 2012. RF Coils for MRI.. Wiley.

von Economo, C.F., 1929. . The cytoarchitectonics of the human cerebral cortex. Humphrey Milford.

Waehnert, M.D., Dinse, J., Weiss, M., Streicher, M.N., Waehnert, P., Geyer, S., Turner, R., Bazin, P.L., 2014. Anatomically motivated modeling of cortical laminae. NeuroImage 93 (Pt 2), 210-220.

Woolrich, M.W., Behrens, T.E., Beckmann, C.F., Jenkinson, M., Smith, S.M., 2004 Multilevel linear modelling for FMRI group analysis using Bayesian inference. NeuroImage 21, 1732-1747.

Woolrich, M.W., Ripley, B.D., Brady, M., Smith, S.M., 2001. Temporal autocorrelation in univariate linear modeling of FMRI data. NeuroImage 14, 1370-1386.

Yacoub, E., Duong, T.Q., Van De Moortele, P.F., Lindquist, M., Adriany, G., Kim, S.G., Ugurbil, K., Hu, X., 2003. Spin-echo fMRI in humans using high spatial resolutions and high magnetic fields. Magn. Reson Med 49, 655-664.

Yacoub, E., Harel, N., Ugurbil, K., 2008. High-field fMRI unveils orientation columns in humans. Proc. Natl. Acad. Sci. U S A 105, 10607-10612.

Yacoub, E., Ugurbil, K., Harel, N., 2006. The spatial dependence of the poststimulus undershoot as revealed by high-resolution BOLD- and CBV-weighted fMRI. J Cereb. Blood Flow. Metab. 26, 634-644.

Yen, C.C.-C., Papoti, D., Silva, A.C., 2017. Investigating the spatiotemporal characteristics of the deoxyhemoglobin-related and deoxyhemoglobin-unrelated functional hemodynamic response across cortical layers in awake marmosets. NeuroImage.

Yushkevich, P.A., Piven, J., Hazlett, H.C., Smith, R.G., Ho, S., Gee, J.C., Gerig, G., 2006. User-guided 3D active contour segmentation of anatomical structures: significantly improved efficiency and reliability. NeuroImage 31, 1116-1128.

Zeng, H., Constable, R.T., 2002. Image distortion correction in EPI: comparison of field mapping with point spread function mapping. Magn. Reson Med 48, 137-146.

Zhao, F., Jin, T., Wang, P., Kim, S.G., 2007. Improved spatial localization of poststimulus BOLD undershoot relative to positive BOLD. NeuroImage 34, 1084-1092.

Zhao, F., Wang, P., Hendrich, K., Ugurbil, K., Kim, S.G., 2006. Cortical layer-dependent BOLD and CBV responses measured by spin-echo and gradient-echo fMRI: insights into hemodynamic regulation. NeuroImage 30, 1149-1160.

Zimmermann, J., Goebel, R., De Martino, F., van de Moortele, P.F., Feinberg, D., Adriany, G., Chaimow, D., Shmuel, A., Ugurbil, K., Yacoub, E., 2011. Mapping the Organization of Axis of Motion Selective Features in Human Area MT Using HighField fMRI. PLoS ONE 6, 1-10. 